\title{
Small Extracellular Vesicles Released from Bioglass/Hydrogel Scaffold Promote Vascularized Bone Regeneration by Transferring miR-23a-3p
}

Hongxing $\mathrm{Hu}$

Tongji University School of Medicine

Hang Zhang

Shanghai Jiaotong University: Shanghai Jiao Tong University

Ziheng Bu

Changhai Hospital

Mingmang Pan

Shanghai Fengxian Central Hospital

Fang Lv

East China Normal University

Zhongtang Liu

Changhai Hospital

liming cheng ( $\square$ limingcheng@tongji.edu.cn )

Tongji University School of Medicine

\section{Research Article}

Keywords: small extracellular vesicles, bone regeneration, angiogenesis, miR-23a-3p, bioglass scaffolds

Posted Date: December 29th, 2021

DOI: https://doi.org/10.21203/rs.3.rs-1158430/v1

License: (9) (i) This work is licensed under a Creative Commons Attribution 4.0 International License.

Read Full License 


\section{Abstract \\ Background}

The treatment of critical-size bone defect is a great difficulty in orthopedics. Osteogenesis and angiogenesis are critical issue during the process of bone repair and remodeling. MSCs-derived small extracellular vesicles (sEVs) show desirable therapeutic prospects in tissue regeneration due to satisfied advantages including high stability, facilitated acquisition and abundant source. However, the effect of Human umbilical cord MSCs-derived sEVs (hUC-MSCs-sEVs) on vascularized bone regeneration and the potential mechanism remains to be investigated. Herein, we aimed to explore the therapeutic effect and the mechanism of hUC-MSCs-sEVs on critical-size bone defect.

\section{Methods}

To investigate the potential osteogenesis and angiogenesis effects of sEVs in vitro, we extracted sEVs from hUC-MSCs, and then SEVs were co-incubated with BMSCs and HUVECs. We next investigated the potential mechanism of $s E V s$ on the effects of osteogenesis and angiogenesis by luciferase reporter gene assay and western blot. We fabricated 3D-printed bioglass scaffold with Gelma/nanoclay hydrogel coatings to load sEVs(BG-gel-sEVs) to ensure in vivo sustained efficacy of sEVs. Finally, the skull defect model was used to evaluate the capacity of vascularized bone regeneration of the composited scaffolds.

\section{Results}

hUC-MSCs-sEVs facilitated calcium deposition and the endothelial network formation, inducing osteogenic differentiation and angiogenesis by delivering miR-23a-3p to activate PTEN/AKT signaling pathway. Additionally, the BG-gel-sEVs composited scaffold achieved vascularized bone regeneration in vivo.

\section{Conclusion}

This finds illuminated that hUC-MSCs-sEVs promoted osteogenesis and angiogenesis by delivering miR23a-3p to activate PTEN/AKT signaling pathway, achieving vascularized bone regeneration.

\section{Introduction}

The treatment of critical-size bone defect occurred by the bone diseases such as osteoporosis, bone infection, osteonecrosis, bone tumor and other diseases is a great difficulty in orthopedics ${ }^{[1]}$. Osteogenesis and angiogenesis are critical issue during the process of bone repair and remodeling. Angiogenesis is an essential process for high-quality bone regeneration due to its critical role in transportation of nutrients, growth factors, metabolic waste, and maintain the internal homeostasis of 
bone issue ${ }^{[2]}$. Currently, the treatments for bone defects include autologous grafts, allografts and bone tissue engineering ${ }^{[3]}$. Autologous grafts have some limitations including secondary injuries, limited quantity of bone and morbidity at the donor site ${ }^{[4]}$. While allografts may have the disadvantage of immunogenic rejection ${ }^{[5]}$. Nowadays, mesenchymal stem cells (MSCs)-based tissue engineering has been considered as an alternative strategy ${ }^{[6,7]}$. However, the application of MSCs-based therapy is limited due to its potential risks including immune rejection and teratoma formation ${ }^{[8,9]}$. Therefore, novel promising strategies to repair critical-size bone defect are desperately needed.

Recent evidence indicated that MSCs-derived exosomes which was largely acquired via the paracrine mechanism have the same therapeutic effect to MSCs-based therapies ${ }^{[10]}$. Human umbilical cord MSCs (hUC-MSCs) show satisfied biological properties, such as rich sources, desirable proliferation capability and facilitated acquisition process ${ }^{[11]}$. Small extracellular vesicles (sEVs) derived MSCs revealed specific advantages than MSCs, including high stability, facilitated acquisition and abundant source ${ }^{[12,13]}$. Previous study reported that human dental pulp stem cells derived small extracellular vesicles (MSCssEVs) promoted bone regeneration ${ }^{[14]}$. However, the effect of hUC-MSCs-derived sEVs (hUC-MSCs-sEVs) on vascularized bone regeneration and the potential mechanism remains to be investigated.

Exosomes are a kind of sEVs with a size of $30-150 \mathrm{~nm}$, containing various proteins, miRNA, ncRNA and growth factors ${ }^{[15-17]}$. MicroRNAs are a kind of small single-strand noncoding RNAs, which mediate target genes silencing by translational repression or mRNA degradation ${ }^{[18,19]}$. Previous studies indicated that $s E V s$ delivered miRNA to mediate intercellular communication and regulate function of recipient cell ${ }^{[20,}$ 21], indicating miRNAs were critical issue in SEVs-mediated cell-to-cell communication. Our group previously demonstrated that hUC-MSCs-SEVs contained abundant miR-23a-3p contributed to repair cartilage through promoting the formation of glycosaminoglycan, extracellular matrix synthesis and collagen deposition ${ }^{[22]}$. However, whether hUC-MSCs-sEVs could enhance angiogenesis and osteogenesis to repair critical-size bone defect by delivering miR-23a-3p remained further researched.

For cartilage ${ }^{[23]}$, skin ${ }^{[24,25]}$ or bone ${ }^{[26]}$ tissue engineering technology, sEVs have been always entrapped into hydrogel to achieve localization and slow-release to enhance vascularization or facilitate tissue regeneration. However, these hydrogels cannot protect some soft tissue like the brain before new bone regeneration due to their weak mechanical properties ${ }^{[27]}$. Additionally, hydrogel with high strength alone fail to bond to the subchondral layer or enhance bone regeneration since high water content and lack of osteo-conduction ${ }^{[5,28]}$. 3D-printed scaffolds with excellent physic-chemical composition and mechanical property are the ideal biomaterial in cell-free bone tissue engineering ${ }^{[4,29]}$. In our previous work, we fabricated Gelatin methacrylate (Gelma)/nanoclay hydrogel with the nice function of sustained release of sEVs ${ }^{[22]}$. Therefore, in this study, we fabricated 3D-printed bioglass (BG) scaffold with Gelma/nanoclay hydrogel coatings to load $s E V s$ to achieve cell-free bone regeneration.

As showed in Scheme 1, in this work, we prepared the BG-gel-sEVs composited scaffold for the repair of calvarial bone defect. The therapeutic sEVs released from BG scaffold with Gelma/nanoclay hydrogel 
coatings were uptaked by BMSCs and endothelial cells. The internalized sEVs facilitated calcium deposition and the endothelial network formation, inducing osteogenic differentiation and angiogenesis. The ideal bone regeneration was achieved by the therapeutic BG-gel-sEVs composited scaffold and this finding may be a potential treatment for the repair of critical-size bone defect.

\section{Materials And Methods \\ 2.1. Cell culture}

hUC-MSCs (Cyagen, USA, HUXUC-01101) were incubated in the medium (Lonza, USA, 12-725F) supplemented serum (Life Science, USA, 259509). The multipotency of hUC-MSCs were verified by inducing hUC-MSCs to osteogenic, chondrogenic, adipogenic cells. The surface marker of MSCs was verified flow cytometry analysis.

\subsection{Isolation and characterization of $\mathrm{sEVs}$}

According to the established protocol[22], sEVs were isolated from the medium of hUC-MSCs. Briefly, hUCMSCs were cultured in serum-free medium for 72 hours. The collected supernatants was centrifuged (300×g for $10 \mathrm{~min}, 2000 \times \mathrm{g}$ for $10 \mathrm{~min}$, and $10000 \times \mathrm{g}$ for $30 \mathrm{~min}$ at $4^{\circ} \mathrm{C}$ ) to removed cell debris and dead cells. Then, the supernatants was transferred to the new ultracentrifuge tube and then centrifuged at $100000 \times \mathrm{g}$ for $70 \mathrm{~min}\left(4^{\circ} \mathrm{C}\right)$. The supernatants were centrifuged again to isolate sEVs.

The size distribution and concentration of sEVs were analyzed by Nanosight NS300 system (Malvern, UK). The morphology of sEVs was identified byTransmission electron microscopy (TEM). The marker proteins were identified through western blotting.

\section{3. $\mathrm{PKH}-26$ staining}

According to the previous protocol[22], sEVs were stained by PKH-26 staining kit. In brief, sEVs was diluted by Dilution $\mathrm{C}$ solution and then sEVs was stained by PKH-26 for 10 min in dark environment. The staining process was terminated by $1 \%$ bovine serum albumin. Then, the mixed solution was centrifuged at $100000 \times \mathrm{g}$ for $70 \mathrm{~min}\left(4^{\circ} \mathrm{C}\right)$. sEVs were obtained by resuspending the deposits with cold PBS. Bone marrow mesenchymal stem cells (BMSCs) were incubated with the labelled sEVs for 24h. Subsequently, $4 \%$ paraformaldehyde (PFA) was used to fix cells, and then the cells were marked with DAPI for $5 \mathrm{~min}$. Fluorescence microscopy was used to visualize the stained cells.

\subsection{Cell proliferation assay}

According to the previous protocol ${ }^{[30]}$, BMSCs were isolated from the femur of 4-6 weeks old female C57BL/ 6 mice. To investigate cell viability, BMSCs were cultured in the a-MEM (contained $10 \%$ fetal bovine serum (Gibco, USA) and $1 \%$ penicillin and streptomycin) supplied sEVs for $24 \mathrm{~h}$ and $48 \mathrm{~h}$. At the point time, according to the manufacturer's instructions, the cells were incubated with cell counting kit-8 (CCK-8, Dojindo, Kumamoto, Japan) for $3 \mathrm{~h}$. OD values of $450 \mathrm{~nm}$ was used to evaluate the cells activity. 


\subsection{ALP activity assay}

As described previously ${ }^{[31]}$, BMSCs were cultured in the osteogenic differentiation medium (a-MEM with ascorbic acid, $1 \mu \mathrm{M}$ dexamethasone, glycerol-2-phosphate, 10\% fetal bovine serum, and $1 \%$ penicillin and streptomycin) contained sEVs. At the point time (7 days), PFA was used to fix cells for $10 \mathrm{~min}$.

Subsequently, the fixed BMSCs were washed by PBS for three times. Then, the cells were cultured with $0.1 \%$ Triton X-100 for 10 min. Furthermore, after incubated with PBST for $10 \mathrm{~min}$, the cells were stained with an ALP kit (Sigma, Germany) at $37^{\circ} \mathrm{C}$ for 30 min.

\subsection{Calcium deposition assay}

BMSCs were seeded in the osteogenic differentiation medium contained SEVs at a density of $1 \times 10^{4}$ cells $/ \mathrm{mL}$ in a 24-well plate for 14 days. At the indicated time, PFA was used to fix cells for $10 \mathrm{~min}$. Subsequently, $\mathrm{ddH}_{2} \mathrm{O}$ were used to rinse cells, and then BMSCs were stained with alizarin red stain (ARS, Sigma, Germany) for 10 min.

\subsection{Wound healing assay}

The effect of sEVs on human umbilical vein endothelial cells (HUVECs) migration was assessed by "wound healing" assay in vitro. In brief, a sterile pipette was used to created "wound" after HUVECs growing in full density in 12 well plates. Then, PBS was used to rinse the cells to remove cell debris. HUVECs were cultured in the fresh medium contained sEVs for $8 \mathrm{~h}$. Subsequently, the medium was removed and then fixed by PFA for $15 \mathrm{~min}$. The cells were stained with $0.2 \%$ crystal violet hydrate solution and then observed using microscope.

\subsection{Tube formation assay}

Matrige ${ }^{\mathrm{TM}}$ matrix (356234, Corning, USA) was used to conduct angiogenesis assay in vitro. According to the previous protocol ${ }^{[32]}$, the plates and sterile pipettes were precooled at $4^{\circ} \mathrm{C}$. $100 \mathrm{ul}$ Matrigel was added into cold 48 -well plates using cold pipettes and then transferred to $37^{\circ} \mathrm{C}$ for solidification. Subsequently, HUVECs cultured on the complete medium contained $s E V s$ were seeded on the solidified Matrigel. After incubation for 8 h, HUVECs were observed under inverted optical microscope. The number of nodes, junctions and branches were calculated by image $\mathrm{J}$ software.

\subsection{Transwell migration assay}

According to the established protocol ${ }^{[33]}$, transwell migration assay was implemented to evaluate the effect of sEVs on migration of BMSCs. In brief, BMSCs incubated in the medium without serum were seeded the upper chamber of transwell system (BD falcon, USA). After $8 \mathrm{~h}$ migration, BMSCs were fixed by $4 \%$ PFA. Then, $0.2 \%$ crystal violet was used to stain the migrated cells, and the cells on the upper chamber were removed. The microscopy was used to visualize the migrated BMSCs.

\subsection{Real-time quantitative polymerase chain reaction (qPCR) assay}


Osteogenic differentiation medium supplemented sEVs was implemented for BMSCs incubation. After incubating for 14 days, TRIzol reagent (Invitrogen Pty Ltd., Australia) was used to extract RNA. RevertAid First Strand cDNA Synthesis Kit (Thermo, USA) was used to achieve RNA reverse-transcribing. The mixture including the CDNA, SYBR, and the primers (OCN, BMP-2, and ALP) was added into the Optical 8Cap strip, and then qPCR) was performed. The primer sequence of above gene was listed in Supplementary table 1.

\subsection{Western blot assay}

Western blot was carried out according to the previous protocol[10]. RIPA was used to harvest the cells, isolating endogenous proteins. BCA protein assay kit (Thermo, USA) was used to detect the concentration of the protein. Subsequently, Equal amounts of proteins were separated by SDS-PAGE gel and then transferred to $0.22 \mu \mathrm{m}$ polyvinylidene difluoride membranes. After being blocking by $5 \%$ fat-free milk, the membranes were incubated with primary antibodies overnight at $4^{\circ} \mathrm{C}$. The membranes were rinsed by TBST and then incubated with secondary antibodies for $1 \mathrm{~h}$ at room temperature. ImmunoStar Western $\mathrm{C}$ (LI-COR, USA) was used to observe the proteins band. The following primary antibodies were used. AntiAkt(CST, USA, 9272, 1:1000), anti-phospho-Akt(CST,USA,9271, 1:1000), anti-PTEN(Promab, China, 20399, 1:1000), anti-GAPDH(CST, USA, 2118, 1:1000), anti- $\beta$-actin(CST,USA,3700, 1:1000), anti-CD63(SBI, USA, EXOAB-CD63A-1, 1:1000), anti-CD81(SBI, USA, EXOAB-CD81A-1, 1:1000), CD9(SBI, USA, EXOAB-CD9A-1, $1: 1000)$.

\subsection{Luciferase reporter assay and transfection}

Lipofectamine 2000 reagent (Invitrogen, USA, 11668019) was used to conduct luciferase reporter assay. According to the manufacturer's instruction, miR-23a-3p mimics and the luciferase report vectors were transfected into HEK-293t cells. Passive lysis buffer was used to cleave the cells after 24 hours transfection. Dual-Luciferase Reporter Assay System (Promega, USA) was used to calculated firefly and renilla luciferase activity.

\subsection{Antagomir-23a-3p treatment}

According to the previous described ${ }^{[22]}$, the cells were cultured with antagomir-23a-3p or scramblemir (Ctrl) in serum-free medium for $6 \mathrm{~h}$. Subsequently, the supernatant was removed and then the cells were incubated in the completed medium. The sequences of antagomir-23a-3p and scramblemir were shown in Supplementary Table 2.

\subsection{Construction and characterization of 3D BG-gel Scaffold}




\subsubsection{Construction of Osteogenic 3D BG/Gel Scaffold}

Bioglass scaffolds were fabricated by digital light processing (DLP) printing technique and sintering process $^{[34]}$. Gelma/nanoclay hydrogel was fabricated as previous described ${ }^{[22]}$. The scaffold was soaked in Gelma/nanoclay hydrogel solution contained sEVs $\left(10 \times 10^{8}\right.$ particles $\left./ \mathrm{mL}\right)$ and then radiated by $365 \mathrm{~nm}$ UV for 2 min to coat sEVs film.

The sEVs released profiles from the scaffolds were calculated by BCA (Thermo, USA) according to the established protocol ${ }^{[25]}$. In brief, the scaffolds were soaked in PBS and the cultured medium was collected at the point time. Then, BCA was used to quantify the profiles of thw released sEVs.

\subsubsection{Scanning electron microscopy (SEM)}

SEM was implemented to observe the structure of the scaffolds and the distribution of $s E V s$ on the scaffolds. The scaffolds coated with sEVs were freeze-dried. After sputter-coated with gold for $30 \mathrm{~s}$, the samples were visualized by SEM.

\subsubsection{Live and dead assay}

As previously described ${ }^{[35]}$, to evaluate the viability of the scaffolds immobilized $s E V s, B M S C s$ were seeded in the scaffold at a density of $1 \times 10^{4}$ cells $/ \mathrm{mL}$ in a 24 -well plate for $24 \mathrm{~h}$. Then, the cells on the scaffolds were stained by Live/Dead staining kit (Invitrogen, USA). The fluorescent images were obtained by fluorescence microscopy.

\subsubsection{Biocompatibility and cytotoxicity assay}

The cytotoxicity of the scaffolds was evaluated using CCK-8 assay. Briefly, $1 \times 10^{4}$ cells $/ \mathrm{mL}$ BMSCs were seeded on the samples and incubated for 1,3 , and 7 days. The viability of BMSCs examined by CCK-8 reflected the cytotoxicity of the scaffold. Data was presented as mean \pm SD of three number of replicates.

\subsection{Animal experiments}

All surgical procedures required the agreement of the ethical review committee of Changhai Hospital, People's Liberation Army Navy Military Medical University. 24 male BALB/C mice (8 weeks old) were anaesthetized by injecting avertin. A sagittal incision of $1.5-2.0 \mathrm{~cm}$ was made on the scalp and then 5 $\mathrm{mm}$ diameter critical-size defect was made on center of cranium by an electric trephine drill (STRONG204). To avoid damage to the dura and brain, the operation procedure was very careful. Then, the scaffolds contained sEVs were implanted into the defect. Moreover, calcein AM (green), alizarin red (red) were injected intramuscularly into these skull defect model mice at 5 and 9 weeks, respectively. 
Additionally, to evaluate the regenerated blood vessel in bone defect region after 12 weeks, mice were perfused with Microfill (MV-122, Flow Tech) after euthanasia, and then the skulls were decalcified and then for micro-CT analysis.

\subsection{Microcomputed tomography (micro-CT)}

The animals were sacrificed at the pointed time (12 weeks post-surgery) and the targeted skulls were collected. The samples were fixed by $4 \%$ paraformaldehyde for $48 \mathrm{~h}$ and rinsed by $\mathrm{dd} \mathrm{H}_{2} \mathrm{O}$. Micro-CT (SkyScan1176, Bruker) were used to evaluate the regeneration of the new bone and blood vessels. The bone and blood vessels were 3D reconstructed by CTvox and then analyzed by CT analysis to obtain the bone tissue volume/total tissue volume (BV/TV), trabecular separation/spacing (Tb.Sp), bone mineral density (BMD) and trabecular thickness (Tb.Th).

\subsection{Histology and immunohistochemical analysis}

The samples were decalcified in EDTA and then dehydrate in a gradient alcohol. After being embedded in paraffin, the skulls were cut into $6 \mu \mathrm{m}$ thickness. Hematoxylin/eosin (HE), masson trichrome and van gieson (VG) staining were performed to estimate the regeneration of the new bone. The regeneration of bone was quantificationally analyzed by ImageJ software. The sequential fluorescent labeling of nondecalcified skull samples was observed under the fluorescent microscope. Immunohistochemical staining was carried out as previously described ${ }^{[36]}$.

\subsection{Statistical analysis}

Quantitative data were presented as the mean \pm standard deviation (SD). Comparation between two group was performed by the unpaired Student's t-test. For the analysis of multiples groups, one-way analysis of variance (ANOVA) was used. Statistical analysis was carried out by Graph pad prism 7. P< 0.05 was considered to be statistically significant.

\section{Results}

\subsection{Identification and Characterizion of hUC-MSCs and hUC-MSCs-sEVs}

When hUC-MSCs were cultured to reach $80 \%$ confluence, the cells showed spindle-like shape under the microscope (Figure 1a). To further follow the criteria of stem cell, flow cytometric was used to analysis the surface marker of cells. As illustrated in Figure.1(b), hUC-MSCs were positive for CD73, CD166, CD105, CD44 and negative for CD45 and CD34. Moreover, hUC-MSCs could be induced to adipogenic, osteogenic and chondrogenic cells (Supplementary Figure 1). As illustrated in Figure.1(c), sEVs showed a 
spherical microvesicle morphology with double membrane structure. Based on measure of nanoparticle tracking analysis, the average diameters of the hUC-MSCs-sEVs were about $155.9 \mathrm{~nm}$ (Figure.1d). Moreover, western blot analysis demonstrated that the extracellular vesicles-related specific marker including CD63, CD81, CD9 were highly expressed on sEVs (Figure 1e). Taken together, the above results indicated that hUC-MSCs-sEVs were successfully isolated from the supernatants of hUC-MSCs.

\section{2. hUC-MSCs-sEVs enhanced the proliferation, migration and osteogenic differentiation of BMSCs}

We used PKH-26 staining kit to label hUC-MSCs-sEVs. Then, sEVs were co-cultured with BMSCs for 24h. As illustrated in Figure 2(a), sEVs stained in red fluorescence were up-taken by BMSCs whose nuclei was stained in blue fluorescence by DAPI. Subsequently, we used CCK-8 assay to measure the viability of BMSCs cultured with different concentration of hUC-MSCs-sEVs (Figure 2b), BMSCs were co-cultured with different concentration of sEVs (sEVs1 and sEVs2 meant that $5 \times 10^{8}$ and $10 \times 10^{8}$ particles $/ \mathrm{mL}$ sEVs respectively). CCK-8 results showed that the number of BMSCs was significantly increased after sEVs treatment, and $\mathrm{sEVs}$ of $10 \times 10^{8}$ particles $/ \mathrm{mL}$ significantly enhanced cells proliferation compared to sEVs of $5 \times 10^{8}$ particles $/ \mathrm{mL}$. Moreover, the transwell migration assay revealed that compared to the control group, the migrated ability of BMSCs on sEVs2 group was significantly increased. The number of migrated BMSCs co-incubated with sEVs of $10 \times 10^{8}$ particles $/ \mathrm{mL}$ was significantly than the $\mathrm{sEV}$ s 1 group (Figure 2c, d). In addition, we used the ALP activity and calcium deposition assay to explore the osteogenic effect of hUC-MSCs-sEVs on BMSCs. Compared to the control and sEVs1 group, the sEVs2 group showed higher ALP positive area, as well as the calcium deposition assay evaluated by ARS staining (Figure 2e). Consistently, the osteogenic-related genes including ALP, bone morphogenetic protein-2(BMP-2), osteocalcin (OCN) were significantly increased after sEVs treatment. The sEVs2 group showed higher upregulation of the osteogenic-related genes compared to other groups (Figure 2f-h).

\subsection{Angiogenetic effect of hUC-MSCs-sEVs in vitro}

PKH-26 staining kit was used to label hUC-MSCs-sEVs. The labelled sEVs in red fluorescence were endocytosed by HUVECs and mainly distributed in the cytoplasm (Figure.3a). Subsequently, we used CCK8 to estimate the effect of hUC-MSCs-sEVs on cell viability. HUVECs were co-incubated with sEVs for $24 \mathrm{~h}$, and the results showed that the number of cells treated with $\mathrm{sEVs}$ of $10 \times 10^{8}$ particles $/ \mathrm{mL}$ was distinctly increased compared to $\mathrm{sEVs}$ of $5 \times 10^{8}$ particles $/ \mathrm{mL}$ and control group (Figure.3b). Moreover, to evaluate the effect of hUC-MSCs-sEVs on migration ability of HUVECs (Figure.3c), we stimulated HUVECs with different concentration of sEVs (sEVs1 and sEVs2 meant that $5 \times 10^{8}$ and $10 \times 10^{8}$ particles $/ \mathrm{mL}$ sEVs respectively) for $8 \mathrm{~h}$. Quantitative analysis indicated that the migration rate of sEVs2 group was significantly higher than other two groups (Figure.3d). Furthermore, matrigel-based assay was used to assess the effect of hUC-MSCs-sEVs on the endothelial network formation ability of HUVECs. As showed in Figure.3(e), the endothelial network formation ability of HUVECs was improved after sEVs treatment, compared to the control group. Quantitative analysis including Nb nodes (Figure.3f), Nb jucntions 
(Figure.3g) and $\mathrm{Nb}$ branches (Figure.3h) revealed that the number of nodes, junctions and branches was highest in sEVs2 group compared to other groups, indicating that $10 \times 10^{8}$ particles $/ \mathrm{mL}$ significantly promoted the endothelial network formation ability of HUVECs.

\subsection{Exosomal miR-23a-3p regulated the expression of PTEN and AKT by targeted 3'UTR of PTEN}

sEVs have been demonstrated to transfer various miRNA to recipient cell to mediate intercellular communication $^{[37]}$. Previous study showed miR-23a-3p was highly expressed in hUC-MSCs-sEVs and demonstrated that $s E V$ s repair cartilage through transferring miR-23a-3p ${ }^{[22]}$. To further investigate the mechanism of exosomal miR-23a-3p in the hUC-MSCs-sEVs promotion of osteogenesis and angiogenesis, microRNA-target-predicting programs was used to predict the potential target genes (Figure.4a). Among the predicted genes, PTEN have been demonstrated to play stimulatory role of osteogenic differentiation of BMSCs ${ }^{[38]}$. To demonstrate PTEN was a putative target of miR-23a-3p, luciferase reporter gene assay was performed. The constructs including WT-psicheck-PTEN and Mutpsicheck-PTEN were transfected into 293 T cells. The decline effect of luciferase activity induced by miR23a-3p was abolished by mutating the binding site of 3'UTR of PTEN (Figure.4b). To fuether investigate whether hUC-MSCs-sEVs promoted osteogenesis and angiogenesis by delivering miR-23a-3p and activating downstream signaling pathways, BMSCs and HUVECs were cultured with sEVs. qPCR results revealed that miR-23a-3p expression was significantly increased in cells co-incubated with SEVs than the cells cultured in absence sEVs (Figure.4c and Supplementary Figure 2). Moreover, western blot results showed that the expression level of T-AKT and P-AKT in cells co-incubated with sEVs were increased, whereas the expression level of PTEN was decreased (Figure.4d and Supplementary Figure 3). Taken together, the above results proved that hUC-MSCs-sEVs promoted osteogenesis and angiogenesis in vitro by delivering miR-23a-3p, and the increased miR-23a-3p suppress the expression of PTEN, and then the inhibitory PTEN activated AKT signaling pathway.

\section{5. miR-23a-3p silencing inhibited hUC-MSCs-sEVs- mediated osteogenesis and pro-angiogenesis in vitro.}

To examine the role of miR-23a-3p in hUC-MSCs-sEVs-mediated osteogenesis and angiogenesis in vitro, antagomirs-23a-3p was used to silence miR-23a-3p expression. We treated BMSCs and HUVECs with antagomirs-23a and then extracted RNA for qPCR analysis. The results showed that miR-23a-3p expression level was significantly decreased in ant-mir-23a groups when compared to control group (Figure 5a and Figure 6a). Subsequently, we evaluated the effect of antagomirs-23a in hUC-MSCs-sEVsmediated osteogenesis and angiogenesis in vitro. As shown in Figure 5 (b) and Figure 6(g), antagomir23a-3p remarkably reduced the cells proliferation induced by hUC-MSCs-sEVs. The number of migrated BMSCs was significantly decreased after antagomirs-23a-3p treatment, indicating that the favorable migration effect induced by sEVs was impaired by antagomirs-23a-3p (Figure 5c, d). In addition, the ALP activity and calcium deposition assay evaluated by ARS staining showed that antagomirs-23a-3p 
reduced the ALP activity induced by hUC-MSCs-sEVs, as well as the calcium deposition assay (Figure 5e). Moreover, antagomirs-23a-3p remarkably decreased the expression of osteogenic-related genes including ALP, OCN and BMP-2 mediated by hUC-MSCs-sEVs (Figure 5f-h)). Additionally, antagomirs-23a-3p reduced the favorable migration and endothelial network formation ability of HUVECs which was induced by sEVs (Figure 6b, d). The quantitative analysis including migrated area analysis (Figure.6c), Nb branches (Figure 6e) and $\mathrm{Nb}$ jucntions (Figure 6f) further supported the above results. Furthermore, the results of western blot showed that when treated antagomir-23a-3p, T-AKT, P-AKT expression level of in cells were decreased, whereas PTEN expression level was increased (Figure $5 i$ and Figure 6h), demonstrating that antagomir-23a-3p impaired sEVs-mediated activation of AKT signaling pathway. Collectively, above results demonstrated that miR-23a-3p silencing attenuated the osteogenic and proangiogenic effect induced by hUC-MSCs-sEVs in vitro.

\subsection{Characterization and release kinetics of engineered BG composited scaffolds.}

SEM confirmed the changes of the surface morphology of the scaffolds (Figure 7a). Live/dead staining assay was performed to investigate the cytotoxic effect of samples. As showed on Figure 7(b), the live BMSCs stained with green fluorescence adhere and survive well on the surface of these scaffolds, and there were few dead cells in red fluorescence, indicating that the scaffolds exhibited favorable cytocompatibility and were suitable for cell growth. To further investigate the sEVs release kinetics in BGsEVs and BG-gel-sEVs scaffolds, the scaffolds were soaked in PBS for 1,3,7 days. At the pointed time, the scaffolds were visualized under the fluorescence microscope. As seen from Figure 7(c), more red dots which meant PKH-26 labeled sEVs were observed on the BG-gel-sEVs scaffolds, compared to that on the BG-sEVs scaffold, indicating that sEVs showed a slow-release profile from the BG-gel-sEVs scaffolds. As seen from Supplementary Figure 4, the OD values was enhanced during the process of culture, indicating that the materials have favorable biocompatibility and without cytotoxicity. Moreover, sEVs were slowreleased when encapsulated into the BG-gel samples (Figure 7d).

\subsection{Effect of BG-gel-sEVs scaffold on bone regeneration in vivo.}

To construct biomimetic structural environment and ensure in vivo sustained efficacy of sEVs for efficient and high-quality bone regeneration, we fabricated 3D-printed bioglass (BG) scaffold with Gelma/nanoclay hydrogel coatings to load sEVs (BG-gel-sEVs) to achieve cell-free bone regeneration.

To address in vivo the potential of BG-gel-sEVs treatment for bone defect, the mice critical calvarial bone defects were established. After 12 weeks of operation, micro-CT was utilized to assess the calvarial bone repair. The re-constructional micro-CT images showed that the defect regions were barely covered by the regenerated bone tissue on $B G$ group, while more newly formed bone tissue were observed in the defect regions on BG-gel-sEVs groups, compared to BG group and BG-gel groups (Figure 8a). Moreover, the 
results of bone volume to tissue volume ratio (BV/TV) revealed that the value on BG-gel-sEVs group was significantly higher than other two groups (Figure 8b). Bone mineral density (BMD), an important index reflecting the quality of the newly formed bone tissue, revealed the same phenomenon with BV/TV (Figure 8c). Additionally, the double fluorescence labeling including calcein and alizarin red was used to further assess bone regeneration. Green calcein labeling and Alizarin red reflected dynamic bone regeneration of 5-8 weeks and 9-12 weeks post-surgery respectively (Figure 8d). As shown in Figure 8(e, f), larger fluorochrome areas of green calcein labeling and Alizarin red labeling were observed on BG-gelsEVs group than other two groups.

Adequate vascular network is essential for the efficient and high-quality bone regeneration. To further evaluate angiogenic effect of BG-gel-sEVs in vivo, microfill was used to reconstruct intracranial vascular after 12 weeks of operation. The regenerated blood vessels in detective regions were shown in Figure 9(a, b). The result revealed that the vascular network regenerated on BG-gel-sEVs group was significantly denser than that on other two groups. BG-gel-sEVs group exhibited the greatest extent of vascularization (Figure 9c). Furthermore, as a specific marker of vein endothelial cells, CD31 was selected to label the newly formed blood vessels in detective region. As shown in Figure 9(d-f), the number and area of CD31 positive vascular on BG-gel-sEVs group was significantly increased compared to BG and BG-gel groups, indicating that hUC-MSCs-sEVs contributed to the regeneration of blood vessels in vivo.

\subsection{Histological evaluation of calvarial bone defects repaired by BG-gel-sEVs scaffolds}

Histological staining was performed to further evaluate the efficacy of hUC-MSCs-sEVs on calvarial bone defects. A mass of fibrous connective tissue which was blue staining by Masson's trichrome staining was filled in the defect area, and few of regenerated bone tissue was presented in BG group. In contrast, the defect region in BG-gel-sEVs group was filled with some newly formed bone tissue with more integrity and maturity which was red staining by Masson's trichrome staining and only a small quality of fibrous connective tissue was observed (Figure 10a, b). Moreover, the undecalcificated bones slicing was stained in the Van Gieson picro-fuchsin staining (Figure 10c), and the results showed that more new bone stained in red staining were formed in the BG-gel-sEVs group than those in the BG and BG-gel group. The immunohistochemical staining was performed to assess the expression of osteocalcin (OCN) in the regenerated bone tissues (Figure 10d). Similar to the results of HE and Masson's trichrome staining, the expression level of OCN in BG-gel-sEVs group was enhanced compared to the BG and BG-gel group, indicating that hUC-MSCs-sEVs contributed to the bone regeneration.

\section{Discussion}

Osteogenesis and angiogenesis are critical factors for the efficient and high-quality bone regeneration ${ }^{[39]}$. Adequate vascular network is able to support the growth and proliferation of the osteoblast by delivering nutrients, oxygen and remove metabolic waste ${ }^{[40,41]}$. Thus, adequate vascular network is the essential for 
achieving vascularized bone regeneration. Currently, various therapies for critical size bone defect have been reported ${ }^{[4-6]}$. However, the application of these therapies was limited due to their own drawbacks ${ }^{[4]}$. Herein, we extracted sEVs from hUC-MSCs, and then sEVs were co-incubated with BMSCs, the results revealed that hUC-MSCs-sEVs remarkably improved the proliferation, migration and osteogenic differentiation of BMSCs by transporting miR-23a-3p. Moreover, hUC-MSCs-sEVs could improve angiogenesis in vitro by enhancing the tube formation ability of HUVECs. Furthermore, we constructed ideal bone-grafting BG-gel scaffold with the favorable mechanical property to loading therapeutic sEVs to achieve their slow release.

hUC-MSCs are ideal cell source for MSCs-based therapy due to the favorable properties including rich sources, desirable proliferation capability and facilitated acquisition process ${ }^{[11]}$. sEVs possess specific advantages than MSCs including high stability, facilitated acquisition and abundant sources ${ }^{[22]}$. Wu and colleagues investigated the therapeutic efficacy of MSC-derived SEVs on OA treatment, and found that $\mathrm{sEVs}$ promoted the chondrogenesis of BMSCs ${ }^{[10]}$. Notably, the stem cells might determine the biological functions of the derived sEVs in turn ${ }^{[42]}$. Recent studies reported that MSCs-sEVs promoted angiogenesis and osteogenesis to achieve vascularized bone regeneration ${ }^{[8,43]}$. Nevertheless, the effect of hUC-MSCs$\mathrm{SEV}$ s on vascularized bone regeneration is still unknow and the potential mechanism remains to be investigated.

Bone repair and remodeling is a complicated process which involves angiogenic and osteogenic interaction ${ }^{[44]}$. Herein, our study showed that hUC-MSCs-sEVs could be uptaken by BMSCs and then facilitated the proliferation, migration of BMSCs, indicating that BMSCs migrated to the defect area and further proliferated which induced by hUC-MSCs-sEVs. Additionally, hUC-MSCs-sEVs promoted the osteogenic differentiation of BMSCs, resulting in alkaline phosphatase (ALP) activation and calcium deposition. ALP activity is considered to the indicator the presence of osteoblast cells and the formation of new bone. The release of calcium ions regulated the activation of osteoblast which was conducive to bone regeneration ${ }^{[45]}$. The above results demonstrated that hUC-MSCs-sEVs were conducive to osteogenesis in vitro. For large segmental bone regeneration, favorable nutrient exchange and adequate oxygen supply transported by vascular network are beneficial for cells migration and distribution, making newly formed bone tissue grows margin-to-center ${ }^{[39]}$. Hu et al demonstrated that BMSCs-derived exosomes promoted the function and angiogenesis ability of HUVECS ${ }^{[17]}$, suggesting that MSC-derived exosomes have great potential on angiogenesis. To further evaluate the function of hUC-MSCs-sEVs on angiogenesis in vitro, HUVECs were co-incubated with hUC-MSCs-sEVs. The results revealed that hUCMSCs-sEVs could enhance the proliferation, migration and tube formation ability of HUVECs, indicating that hUC-MSCs-sEVs could regulate the function and activity of HUVECs, improving angiogenesis in vitro. Consistently, hUC-MSCs-sEVs possess great therapeutic potential in critical size bone defect due to the ability of coupling osteogenesis and angiogenesis.

As the carriers produced by derived cells, sEVs delivered bioactive ingredient including nucleic acids, proteins, lipids and other signaling molecules to recipient cells, facilitating intercellular 
communication ${ }^{[46]}$. MiRNA, a class of small noncoding RNA, plays critical regulatory role in gene expression by directly modifying mRNA at post-transcriptional level ${ }^{[47,48]}$. Recent studies have reported that exosomal miRNAs played essential regulatory role in bone and vascular homoeostasis ${ }^{[49,50]}$. In previous study, we demonstrated that miR-23a-3p was highly expressed in hUC-MSCs-sEVs ${ }^{[22]}$. While, the role of exosomal miR-23a-3p in osteogenesis and angiogenesis remain elusive. Thus, to further investigate the role of exosomal miR-23a-3p in BMSCs and HUVECs, we co-cultured sEVs with cells and found that the expression of miR-23a-3p in BMSCs and HUVECs was increased. Moreover, luciferase assay showed that miR-23a-3p mimic down-regulated the expression of PTEN, whereas miR-23a-3p mutant promoted the production of PTEN, indicating that miR-23a-3p bound the 3'UTR of PTEN, resulting in the down regulation of PTEN. Previous study showed that PTEN played negative regulated role in AKT signaling pathway ${ }^{[51]}$. The activation of PTEN/AKT signaling pathway promoted BMSCs osteogenic differentiation ${ }^{[38]}$. In this study, we demonstrated that hUC-MSCs-SEVs promoted angiogenesis and osteogenesis in vitro by delivering miR-23a-3p. The up-regulated miR-23a-3p in cells targeted mRNA of PTEN and suppressed the expression of PTEN, resulting in the activation of AKT signaling pathway. Additionally, the downregulated miR-23a-3p in cells induced by antogomirs-23a weaken the effect of angiogenesis and osteogenesis which induced by hUC-MSCs-sEVs. In general, hUC-MSCs-sEVs may be a promising therapeutic treatment for critical size bone defect.

Bioglass scaffolds with excellent mechanical, osteo-inductive and controlled degradation property are the ideal biomaterial in cell-free bone tissue engineering ${ }^{[1,4,29]}$. Previous study revealed that hUC-MSCs-sEVs entrapped into Gelma/nanoclay hydrogel were release in sustained manner through the controlled degradation of hydrogel ${ }^{[22]}$. Herein, we fabricated BG scaffold with Gelma/nanoclay hydrogel coatings to load SEVs to achieve vascularized bone regeneration. In vivo results showed that BG-gel-sEVs composited scaffold effectively improved angiogenesis and osteogenesis, repairing skull bone defect.

\section{Conclusions}

In general, our study found that hUC-MSCs-sEVs effectively improved angiogenesis and osteogenesis by delivering miR-23a-3p to activate PTEN/AKT signaling pathway. Additionally, the BG-gel-sEVs composited scaffold achieved vascularized bone regeneration by slowly releasing sEVs. Consistently, the BG-gel-sEVs composited scaffold has essential potential in skull bone defect treatment.

\section{Abbreviations}

MSCs: Mesenchymal stem cells

hUC-MSCs: Human umbilical cord mesenchymal stem cells

hUC-MSCs-sEVs: Human umbilical cord mesenchymal stem cells derived small extracellular vesicles

BG: Bioglass

Page $14 / 31$ 
BG-gel-sEVs: Bioglass scaffold with Gelma/nanoclay hydrogel

Gelma: Gelatin methacrylate

BMP-2: Bone morphogenetic protein-2

OCN: Osteocalcin

ALP: Alkaline phosphatase

HUVECs: Human umbilical vein endothelial cells

Micro-CT: Microcomputed tomography

BV/TV: Bone tissue volume/total tissue volume

Tb.Sp: Trabecular separation/spacing

BMD: Bone mineral density

Tb.Th: Trabecular thickness

HE: Hematoxylin/eosin

TEM: Transmission electron microscopy

\section{Declarations}

\section{Ethics approval and consent to participate}

All animal experiments were performed in accordance with the guidelines and ethical procedures of the ethical review committee of Changhai Hospital, People's Liberation Army Navy Military Medical University.

\section{Consent for publication}

Not applicable.

\section{Availability of data and materials}

The datasets used and/or analyzed during the current study are available from the corresponding author on reasonable request.

\section{Funding}

This work was supported by the National Natural Science Foundation of China (81802202ð51673212) 


\section{Authors' contributions}

Fang Lv, ZhongtangLiu and Liming Cheng designed and conceived the study. Hongxing Hu and Hang Zhang performed the experiments and wrote the manuscript. Mingmang Pan edited and reviewed the manuscript, Ziheng Bu provided experimental equipment. All authors have read and agreed to the published version of the manuscript.

\section{Acknowledgements}

We would like to thank Xinlu Liu for Scheme drawing of the manuscript.

\section{Competing interests}

The authors declare no potential conflict of interest.

\section{References}

[1] R. Chen, J. Wang, C. S. Liu. Biomaterials Act as Enhancers of Growth Factors in Bone Regeneration. Adv Funct Mater, 2016, 26(48): 8810-8823

[2] G. Mao, Z. Zhang, S. Hu, Z. Zhang, Z. Chang, Z. Huang, W. Liao, Y. Kang. Exosomes derived from miR92a-3p-overexpressing human mesenchymal stem cells enhance chondrogenesis and suppress cartilage degradation via targeting WNT5A. Stem cell research \& therapy, 2018, 9(1): 247

[3] B. Aldemir Dikici, G. C. Reilly, F. Claeyssens. Boosting the Osteogenic and Angiogenic Performance of Multiscale Porous Polycaprolactone Scaffolds by In Vitro Generated Extracellular Matrix Decoration. ACS Appl Mater Interfaces, 2020, 12(11): 12510-12524

[4] C. Garot, G. Bettega, C. Picart. Additive Manufacturing of Material Scaffolds for Bone Regeneration: Toward Application in the Clinics. Adv Funct Mater, 2021, 31(5):

[5] J. H. Huang, X. M. Yin, Y. Xu, C. C. Xu, X. Lin, F. B. Ye, Y. Cao, F. Y. Lin. Systemic Administration of Exosomes Released from Mesenchymal Stromal Cells Attenuates Apoptosis, Inflammation, and Promotes Angiogenesis after Spinal Cord Injury in Rats. Journal of neurotrauma, 2017, 34(24): 33883396

[6] P. Chocholata, V. Kulda, V. Babuska. Fabrication of Scaffolds for Bone-Tissue Regeneration. Materials, 2019, 12(4):

[7] Zhipo Du, Xinxing Feng, Guangxiu Cao, Zhending She, Rongwei Tan, Katerina E. Aifantis, Ruihong Zhang, Xiaoming Li. The effect of carbon nanotubes on osteogenic functions of adipose-derived mesenchymal stem cells in vitro and bone formation in vivo compared with that of nano-hydroxyapatite and the possible mechanism. Bioactive Materials, 2021, 6(2): 333-345 
[8] Y. Zha, Y. W. Li, T. Y. Lin, J. Chen, S. M. Zhang, J. L. Wang. Progenitor cell-derived exosomes endowed with VEGF plasmids enhance osteogenic induction and vascular remodeling in large segmental bone defects. Theranostics, 2021, 11(1): 397-409

[9] H. Xing, Z. Zhang, Q. Mao, C. Wang, Y. Zhou, X. Zhou, L. Ying, H. Xu, S. Hu, N. Zhang. Injectable exosome-functionalized extracellular matrix hydrogel for metabolism balance and pyroptosis regulation in intervertebral disc degeneration. J Nanobiotechnology, 2021, 19(1): 264

[10] J. Wu, L. Kuang, C. Chen, J. Yang, W. N. Zeng, T. Li, H. Chen, S. Huang, Z. Fu, J. Li, R. Liu, Z. Ni, L. Chen, L. Yang. miR-100-5p-abundant exosomes derived from infrapatellar fat pad MSCs protect articular cartilage and ameliorate gait abnormalities via inhibition of mTOR in osteoarthritis. Biomaterials, 2019, 206: $87-100$

[11] W. Yang, J. Zhang, B. Xu, Y. He, W. Liu, J. Li, S. Zhang, X. Lin, D. Su, T. Wu, J. Li. HucMSC-Derived Exosomes Mitigate the Age-Related Retardation of Fertility in Female Mice. Mol Ther, 2020, 28(4): 12001213

[12] M. Wang, C. Wang, M. Chen, Y. Xi, W. Cheng, C. Mao, T. Xu, X. Zhang, C. Lin, W. Gao, Y. Guo, B. Lei. Efficient Angiogenesis-Based Diabetic Wound Healing/Skin Reconstruction through Bioactive Antibacterial Adhesive Ultraviolet Shielding Nanodressing with Exosome Release. ACS nano, 2019:

[13] Z. G. Zhang, B. Buller, M. Chopp. Exosomes - beyond stem cells for restorative therapy in stroke and neurological injury. Nature reviews Neurology, 2019, 15(4): 193-203

[14] W. B. Swanson, Z. Zhang, K. M. Xiu, T. Gong, M. Eberle, Z. Q. Wang, P. X. Ma. Scaffolds with controlled release of pro-mineralization exosomes to promote craniofacial bone healing without cell transplantation. Acta Biomaterialia, 2020, 118: 215-232

[15] R. Kalluri, V. S. LeBleu. The biology, function, and biomedical applications of exosomes. Science, 2020, 367(6478):

[16] C. A. Fitts, N. Ji, Y. Li, C. Tan. Exploiting Exosomes in Cancer Liquid Biopsies and Drug Delivery. Adv Healthc Mater, 2019, 8(6): e1801268

[17] Y. Q. Hu, R. Y. Tao, L. Chen, Y. Xiong, H. Xue, L. C. Hu, C. C. Yan, X. D. Xie, Z. Lin, A. C. Panayi, B. B. Mi, G. H. Liu. Exosomes derived from pioglitazone-pretreated MSCs accelerate diabetic wound healing through enhancing angiogenesis. J Nanobiotechnol, 2021, 19(1):

[18] C. J. Li, P. Cheng, M. K. Liang, Y. S. Chen, Q. Lu, J. Y. Wang, Z. Y. Xia, H. D. Zhou, X. Cao, H. Xie, E. Y. Liao, X. H. Luo. MicroRNA-188 regulates age-related switch between osteoblast and adipocyte differentiation. The Journal of clinical investigation, 2015, 125(4): 1509-1522

[19] V. K. Mayya, M. N. Flamand, A. M. Lambert, S. M. Jafarnejad, J. A. Wohlschlegel, N. Sonenberg, T. F. Duchaine. microRNA-mediated translation repression through GYF-1 and IFE-4 in C. elegans development. 
Nucleic Acids Res, 2021, 49(9): 4803-4815

[20] A. Thind, C. Wilson. Exosomal miRNAs as cancer biomarkers and therapeutic targets. J Extracell Vesicles, 2016, 5:

[21] Z. S. Gao, C. J. Zhang, N. Xia, H. Tian, D. Y. Li, J. Q. Lin, X. F. Mei, C. Wu. Berberine-loaded M2 macrophage-derived exosomes for spinal cord injury therapy. Acta Biomater, 2021, 126: 211-223

[22] H. Hu, L. Dong, Z. Bu, Y. Shen, J. Luo, H. Zhang, S. Zhao, F. Lv, Z. Liu. miR-23a-3p-abundant small extracellular vesicles released from Gelma/nanoclay hydrogel for cartilage regeneration. J Extracell Vesicles, 2020, 9(1): 1778883

[23] P. Chen, L. Zheng, Y. Wang, M. Tao, Z. Xie, C. Xia, C. Gu, J. Chen, P. Qiu, S. Mei, L. Ning, Y. Shi, C. Fang, S. Fan, X. Lin. Desktop-stereolithography 3D printing of a radially oriented extracellular matrix/mesenchymal stem cell exosome bioink for osteochondral defect regeneration. Theranostics, 2019, 9(9): 2439-2459

[24] S. C. Tao, S. C. Guo, M. Li, Q. F. Ke, Y. P. Guo, C. Q. Zhang. Chitosan Wound Dressings Incorporating Exosomes Derived from MicroRNA-126-Overexpressing Synovium Mesenchymal Stem Cells Provide Sustained Release of Exosomes and Heal Full-Thickness Skin Defects in a Diabetic Rat Model. Stem Cells Transl Med, 2017, 6(3): 736-747

[25] M. Wang, C. G. Wang, M. Chen, Y. W. Xi, W. Cheng, C. Mao, T. Z. Xu, X. X. Zhang, C. Lin, W. Y. Gao, Y. Guo, B. Lei. Efficient Angiogenesis-Based Diabetic Wound Healing/Skin Reconstruction through Bioactive Antibacterial Adhesive Ultraviolet Shielding Nanodressing with Exosome Release. Acs Nano, 2019, 13(9): 10279-10293

[26] S. Chen, Y. Tang, Y. Liu, P. Zhang, L. Lv, X. Zhang, L. Jia, Y. Zhou. Exosomes derived from miR-375overexpressing human adipose mesenchymal stem cells promote bone regeneration. Cell Prolif, 2019, 52(5): e12669

[27] B. Xu, P. B. Zheng, F. Gao, W. Wang, H. T. Zhang, X. R. Zhang, X. Q. Feng, W. G. Liu. A Mineralized High Strength and Tough Hydrogel for Skull Bone Regeneration. Adv Funct Mater, 2017, 27(4):

[28] T. Nonoyama, S. Wada, R. Kiyama, N. Kitamura, M. T. Mredha, X. Zhang, T. Kurokawa, T. Nakajima, Y. Takagi, K. Yasuda, J. P. Gong. Double-Network Hydrogels Strongly Bondable to Bones by Spontaneous Osteogenesis Penetration. Adv Mater, 2016, 28(31): 6740-6745

[29] M. M. Zhai, Y. Zhu, M. Y. Yang, C. B. Mao. Human Mesenchymal Stem Cell Derived Exosomes Enhance Cell-Free Bone Regeneration by Altering Their miRNAs Profiles. Adv Sci, 2020, 7(19):

[30] C. Wang, H. Hu, Z. Li, Y. Shen, Y. Xu, G. Zhang, X. Zeng, J. Deng, S. Zhao, T. Ren, Y. Zhang. Enhanced Osseointegration of Titanium Alloy Implants with Laser Microgrooved Surfaces and Graphene Oxide Coating. ACS Appl Mater Interfaces, 2019, 11(43): 39470-39483 
[31] C. Lin, L. Liu, C. Zeng, Z. K. Cui, Y. Chen, P. Lai, H. Wang, Y. Shao, H. Zhang, R. Zhang, C. Zhao, H. Fang, D. Cai, X. Bai. Activation of mTORC1 in subchondral bone preosteoblasts promotes osteoarthritis by stimulating bone sclerosis and secretion of CXCL12. Bone research, 2019, 7: 5

[32] L. Liu, Y. Liu, C. Feng, J. Chang, R. Fu, T. Wu, F. Yu, X. Wang, L. Xia, C. Wu, B. Fang. Lithium-containing biomaterials stimulate bone marrow stromal cell-derived exosomal miR-130a secretion to promote angiogenesis. Biomaterials, 2019, 192: 523-536

[33] Z. Zhu, Y. Liu, Y. Xue, X. Cheng, W. Zhao, J. Wang, R. He, Q. Wan, X. Pei. Tazarotene Released from Aligned Electrospun Membrane Facilitates Cutaneous Wound Healing by Promoting Angiogenesis. ACS Appl Mater Interfaces, 2019, 11(39): 36141-36153

[34] F. Baino, G. Magnaterra, E. Fiume, A. Schiavi, L. P. Tofan, M. Schwentenwein, E. Verne. Digital light processing stereolithography of hydroxyapatite scaffolds with bone-like architecture, permeability, and mechanical properties. J Am Ceram Soc, 2021:

[35] L. Wei, S. Wu, M. Kuss, X. Jiang, R. Sun, P. Reid, X. Qin, B. Duan. 3D printing of silk fibroin-based hybrid scaffold treated with platelet rich plasma for bone tissue engineering. Bioact Mater, 2019, 4: 256260

[36] Y. Chen, T. Wu, S. Huang, C. W. Suen, X. Cheng, J. Li, H. Hou, G. She, H. Zhang, H. Wang, X. Zheng, Z. Zha. Sustained Release SDF-1alpha/TGF-beta1-Loaded Silk Fibroin-Porous Gelatin Scaffold Promotes Cartilage Repair. ACS Appl Mater Interfaces, 2019, 11(16): 14608-14618

[37] T. J. DiStefano, K. Vaso, G. Danias, H. N. Chionuma, J. R. Weiser, J. C. latridis. Extracellular Vesicles as an Emerging Treatment Option for Intervertebral Disc Degeneration: Therapeutic Potential, Translational Pathways, and Regulatory Considerations. Advanced Healthcare Materials, 2021:

[38] C. Y. Jin, L. F. Jia, Z. H. Tang, Y. F. Zheng. Long non-coding RNA MIR22HG promotes osteogenic differentiation of bone marrow mesenchymal stem cells via PTEN/AKT pathway. Cell Death Dis, 2020, 11(7):

[39] X. Han, M. Sun, B. Chen, Q. Saiding, J. Zhang, H. Song, L. Deng, P. Wang, W. Gong, W. Cui. Lotus seedpod-inspired internal vascularized 3D printed scaffold for bone tissue repair. Bioact Mater, 2021, 6(6): $1639-1652$

[40] L. F. Frohlich. MicroRNAs at the Interface between Osteogenesis and Angiogenesis as Targets for Bone Regeneration. Cells-Basel, 2019, 8(2):

[41] M. J. Chen, Y. H. Zhang, W. A. Zhang, J. Li. Polyhedral Oligomeric Silsesquioxane-Incorporated Gelatin Hydrogel Promotes Angiogenesis during Vascularized Bone Regeneration. Acs Appl Mater Inter, 2020, 12(20): 22410-22425 
[42] H. Jing, X. Zhang, K. Luo, Q. Luo, M. Yin, W. Wang, Z. Zhu, J. Zheng, X. He. miR-381-abundant small extracellular vesicles derived from kartogenin-preconditioned mesenchymal stem cells promote chondrogenesis of MSCs by targeting TAOK1. Biomaterials, 2020, 231: 119682

[43] L. Liu, F. Yu, L. Li, L. Zhou, T. Zhou, Y. Xu, K. Lin, B. Fang, L. Xia. Bone marrow stromal cells stimulated by strontium-substituted calcium silicate ceramics: release of exosomal miR-146a regulates osteogenesis and angiogenesis. Acta Biomater, 2021, 119: 444-457

[44] X. T. Wang, X. L. Li, J. Li, L. D. Zhai, D. Q. Liu, A. Abdurahman, Y. F. Zhang, H. Yokota, P. Zhang. Mechanical loading stimulates bone angiogenesis through enhancing type $\mathrm{H}$ vessel formation and downregulating exosomal miR-214-3p from bone marrow-derived mesenchymal stem cells. Faseb J, 2021, 35(1):

[45] J. Jeong, J. H. Kim, J. H. Shim, N. S. Hwang, C. Y. Heo. Bioactive calcium phosphate materials and applications in bone regeneration. Biomater Res, 2019, 23(1):

[46] Y. Nakao, T. Fukuda, Q. Zhang, T. Sanui, T. Shinjo, X. Kou, C. Chen, D. Liu, Y. Watanabe, C. Hayashi, H. Yamato, K. Yotsumoto, U. Tanaka, T. Taketomi, T. Uchiumi, A. D. Le, S. Shi, F. Nishimura. Exosomes from TNF-alpha-treated human gingiva-derived MSCs enhance M2 macrophage polarization and inhibit periodontal bone loss. Acta Biomater, 2021, 122: 306-324

[47] Y. Li, C. Fan, L. Wang, T. Lan, R. Gao, W. Wang, S. Y. Yu. MicroRNA-26a-3p rescues depression-like behaviors in male rats via preventing hippocampal neuronal anomalies. J Clin Invest, 2021:

[48] Y. Ito, T. Matsuzaki, F. Ayabe, S. Mokuda, R. Kurimoto, T. Matsushima, Y. Tabata, M. Inotsume, H. Tsutsumi, L. Liu, M. Shinohara, Y. Tanaka, R. Nakamichi, K. Nishida, M. K. Lotz, H. Asahara. Both microRNA-455-5p and $-3 p$ repress hypoxia-inducible factor-2alpha expression and coordinately regulate cartilage homeostasis. Nat Commun, 2021, 12(1): 4148

[49] A. Liu, D. Lin, H. Zhao, L. Chen, B. Cai, K. Lin, S. G. Shen. Optimized BMSC-derived osteoinductive exosomes immobilized in hierarchical scaffold via lyophilization for bone repair through Bmpr2/Acvr2b competitive receptor-activated Smad pathway. Biomaterials, 2021, 272: 120718

[50] S. Aday, I. Hazan-Halevy, A. Chamorro-Jorganes, M. Anwar, M. Goldsmith, N. Beazley-Long, S. Sahoo, N. Dogra, W. Sweaad, F. Catapano, S. Ozaki-Tan, G. D. Angelini, P. Madeddu, A. V. Benest, D. Peer, C. Emanueli. Bioinspired artificial exosomes based on lipid nanoparticles carrying let-7b-5p promote angiogenesis in vitro and in vivo. Mol Ther, 2021, 29(7): 2239-2252

[51] Brendan D. Manning, Alex Toker. AKT/PKB Signaling: Navigating the Network. Cell, 2017, 169(3): 381405

\section{Scheme}




\section{Figures}

a

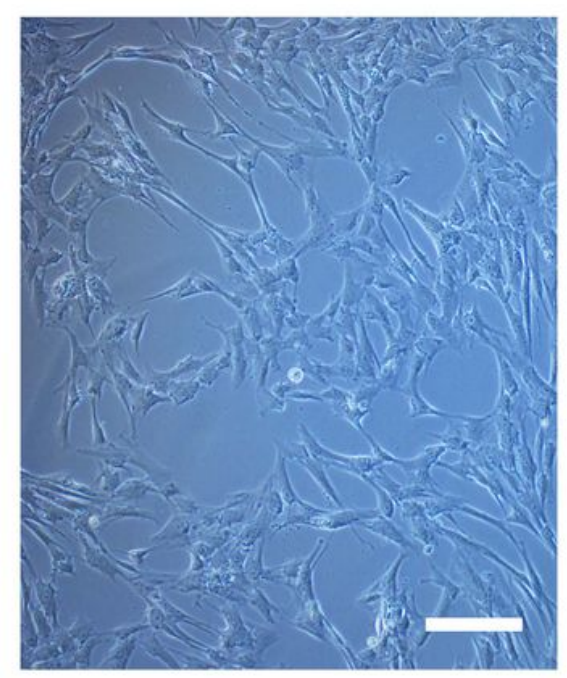

C

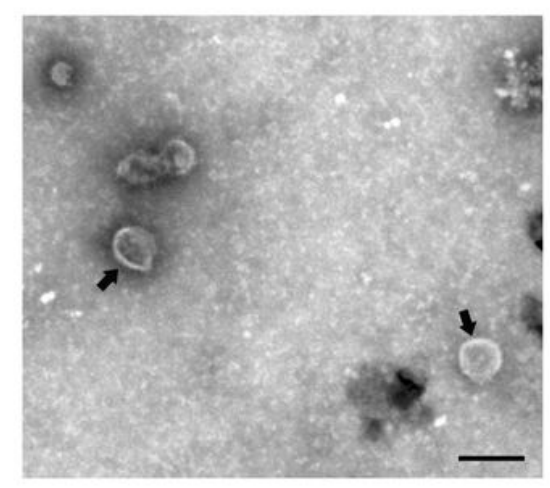

b
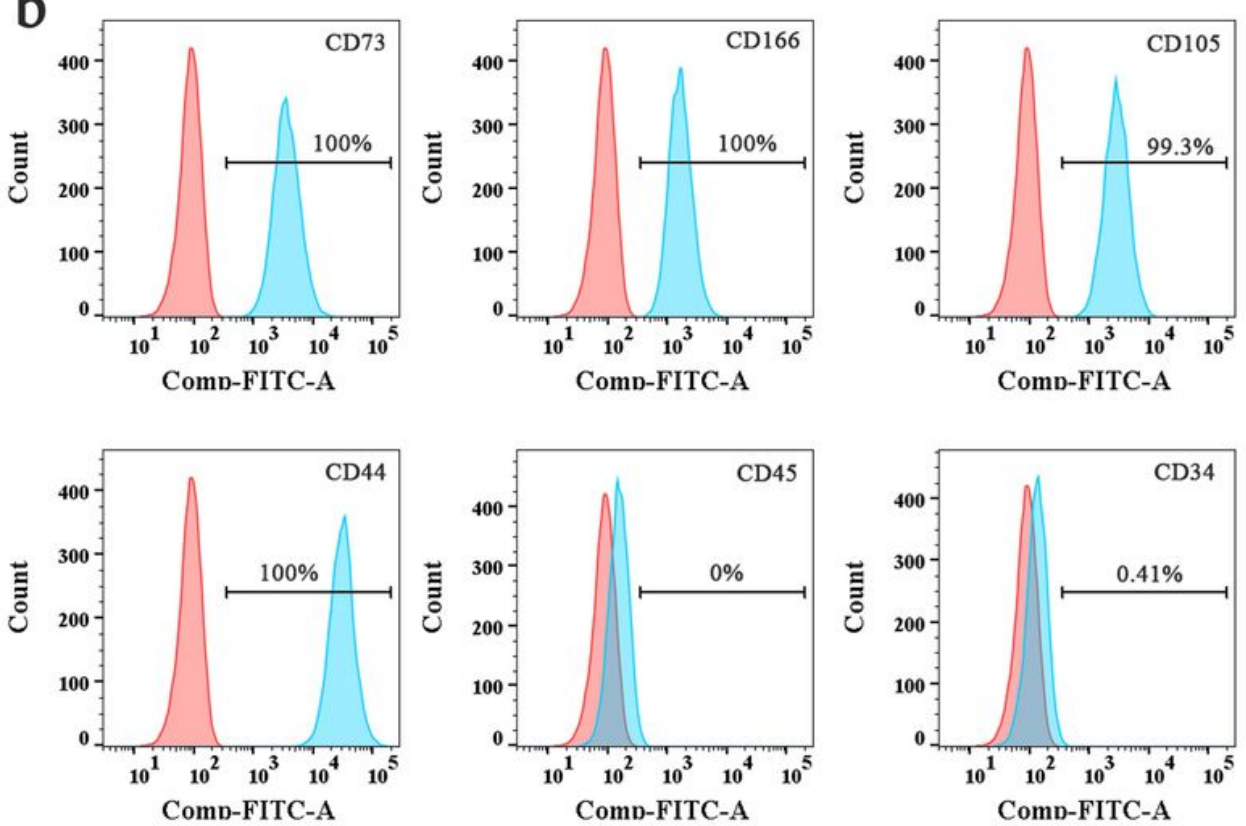

d

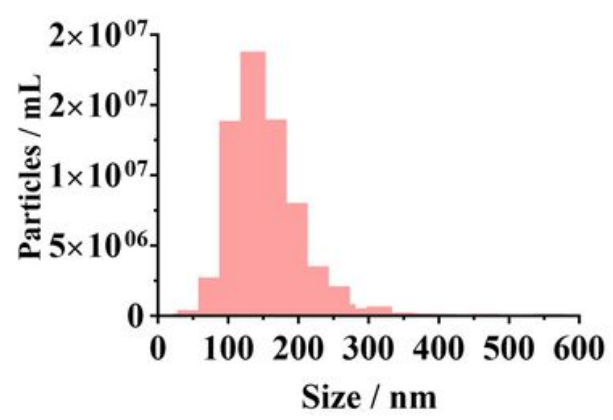

e

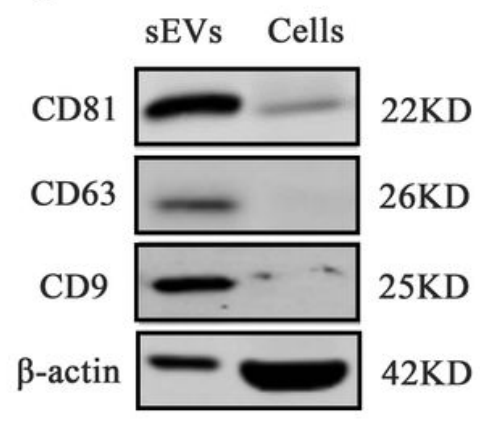

\section{Figure 1}

Characterizion of hUC-MSCs and hUC-MSCs-sEVs. (a) hUC-MSCs exhibited spindle-like morphology. Scale bar: $100 \mathrm{~nm}$. (b) Flow cytometric analysis of surface marker of hUC-MSCs. The red filled curves mean isotype control and the blue filled curves represent the surface marker of cells. (c)The morphology of hUCMSCs-sEVs observed under transmission electron microscopy (TEM). Scale bar: 200nm. (d) Nanoparticle tracking analysisshow the particle size of hUC-MSCs-sEVs. (e) The positive surface markers of hUC-MSCs including CD9, CD63 and CD81 were determined by western blot. 
a

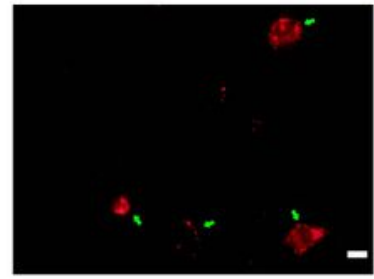

PKH-26

c

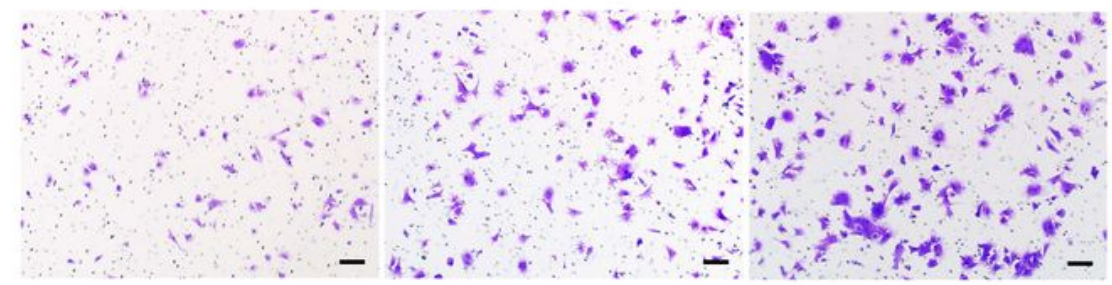

Ctrl

e

ALP

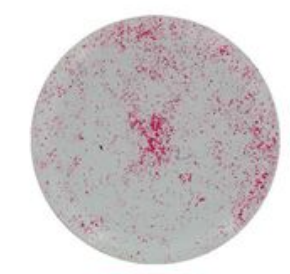

ARS

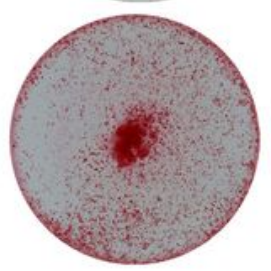

Ctrl
sEVs1

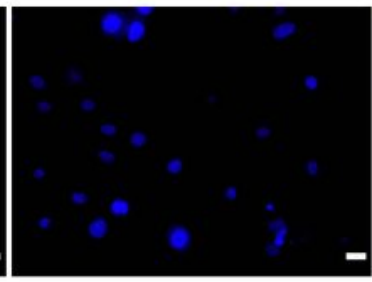

DAPI

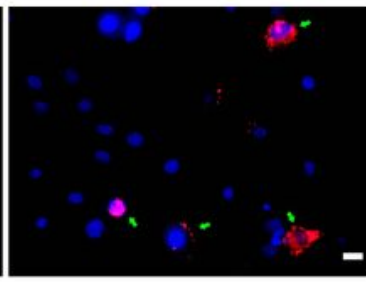

Merge

sEVs2
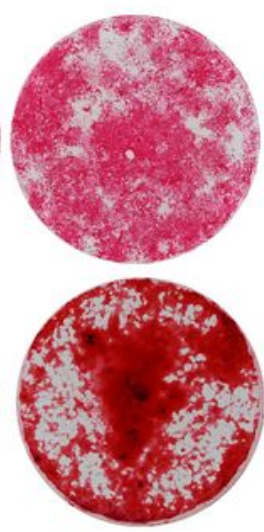

sEVs2 b

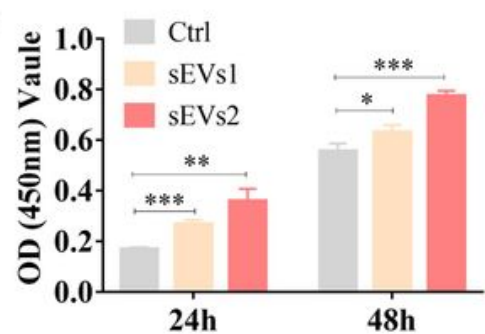

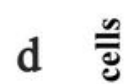

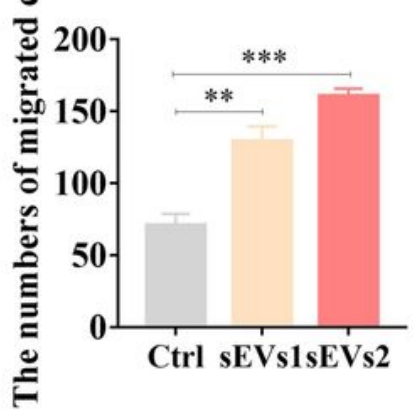

g

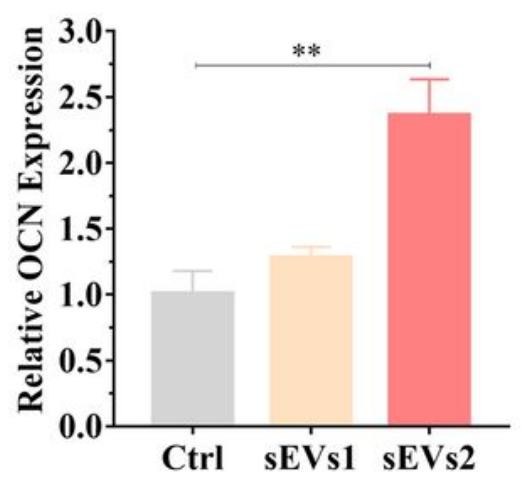

f

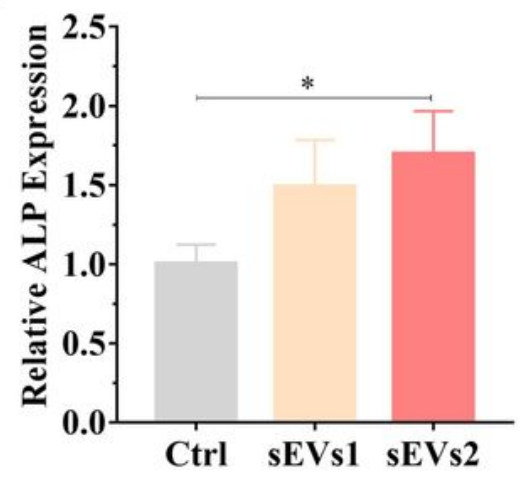

h

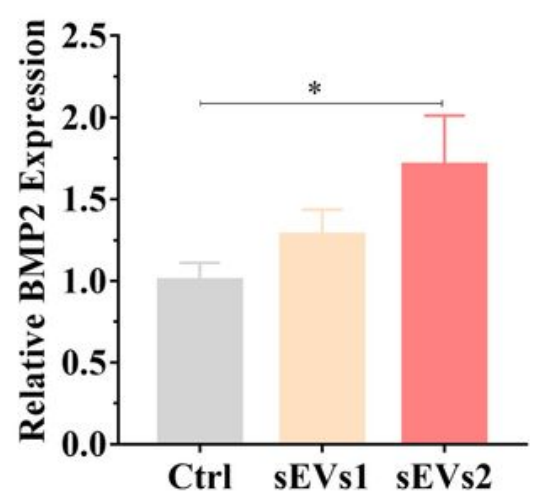

\section{Figure 2}

hUC-MSCs-sEVsenhanced the proliferation, migration and osteogenic differentiation of BMSCs.

(a)Fluorescent micrograph of sEVs labeled by PKH-26.BMSCs were cultured with the labelled sEVs for 24 h. Scale bar:20um.(b) CCK-8 assay of BMSCs cultured with sEVs. ${ }^{*} P<0.05, * * P<0.01, * * * P<0.001$. (c) Transwellassay of BMSCs cultured with sEVs for 8h. Scale bar: 50um. (d) Quantitative analysis of the number of migrated BMSCs. ${ }^{* * P}<0.01,{ }^{*} * \mathrm{P}<0.001$. (e) Alkaline phosphatase (ALP) activity and Alizarin 
Red S (ARS) staining of BMSCs. (f-h)The osteogenic-related gene of BMSCs cultured with different concentration of sEVswas measured via the qPCR, including OCN, BMP-2 and ALP.

a

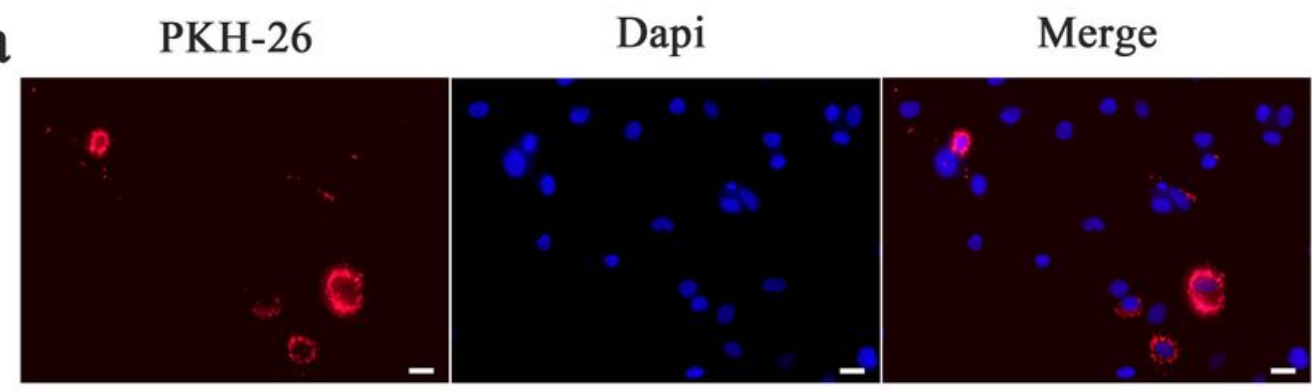

b

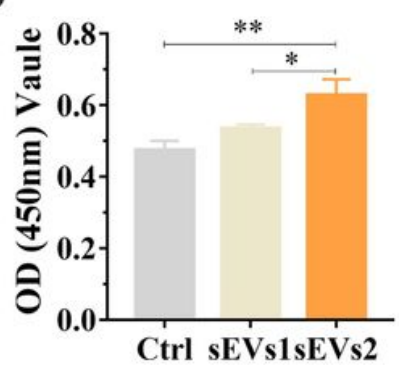

C

Ctrl

sEVs1

sEVs2

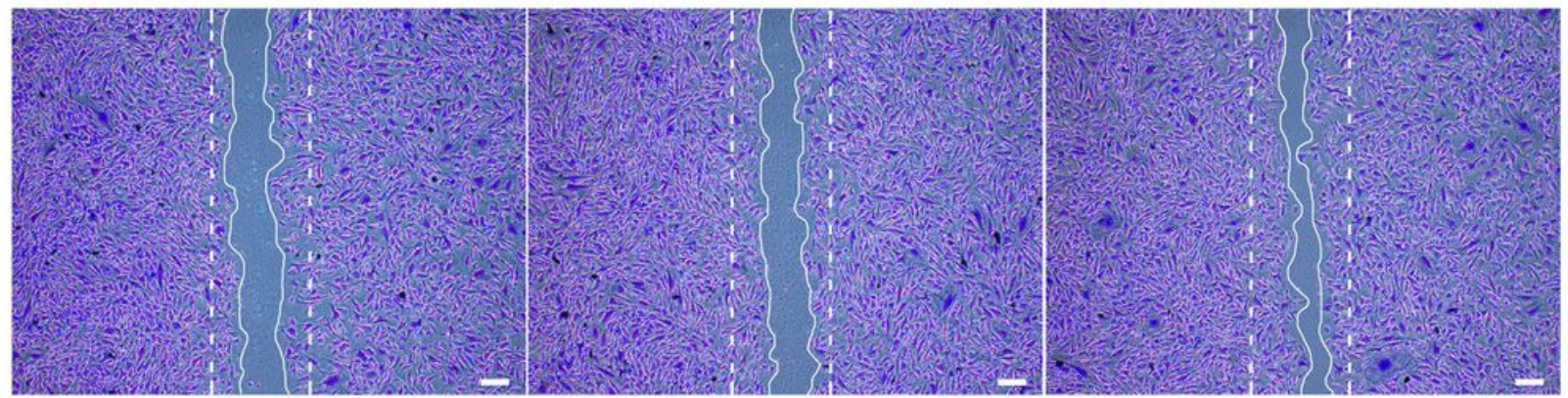

d

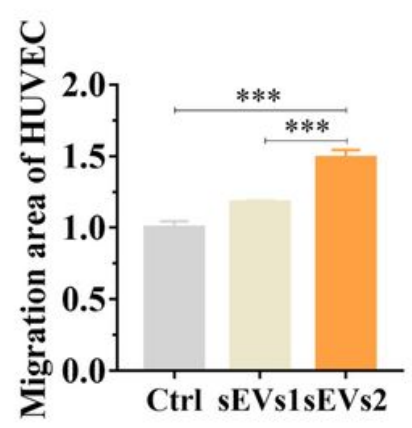

e

f

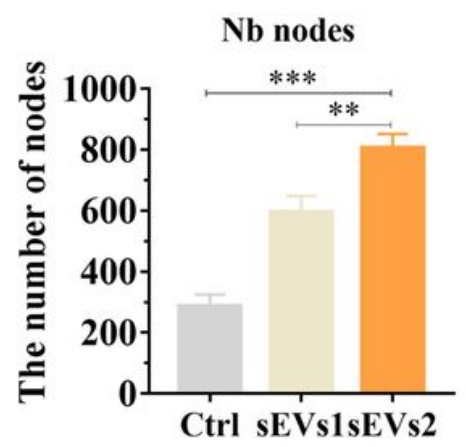

g

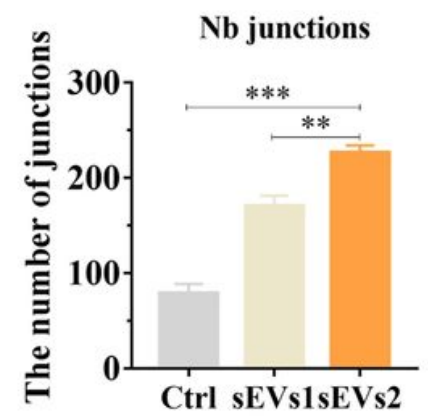

sEVs2

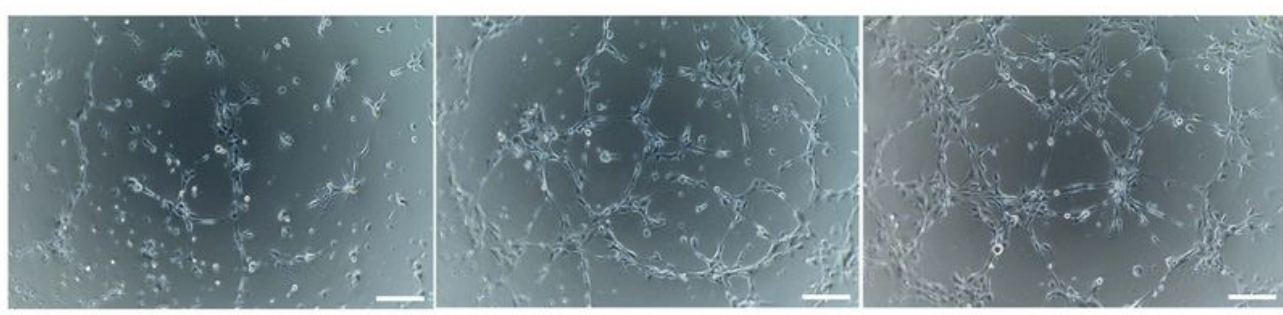

h

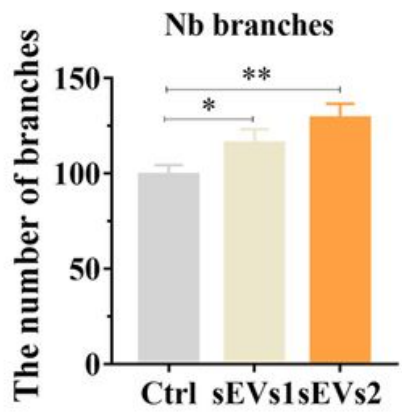

Figure 3

Angiogenetic effect of hUC-MSCs-sEVs in vitro. (a) Fluorescent micrograph of sEVs labeled by PKH26.HUVECs were cultured with the labelled sEVs for $24 \mathrm{~h}$. Scale bar:20um. (b) CCK-8 assay of HUVECs cultured with sEVs for $24 \mathrm{~h}$. ${ }^{*} \mathrm{P}<0.05$, ${ }^{\star *} \mathrm{P}<0.01$. (c)Representative images of "wound healing" assay of HUVECs after $8 \mathrm{~h}$ co-culture with hUC-MSCs-sEVs and (d) Quantitative analysis of the relative migrated 
percentage of HUVECs in different groups. (e) Matrigel-based assay for HUVECs cultured with hUC-MSCssEVs. Scale bar: 100um. (f) Nb nodes, (g) Nb junctions, (h)Nb branches were all analyzed about tube formation assay by Image $\mathrm{J}$.

a

Bioinformatics analysis

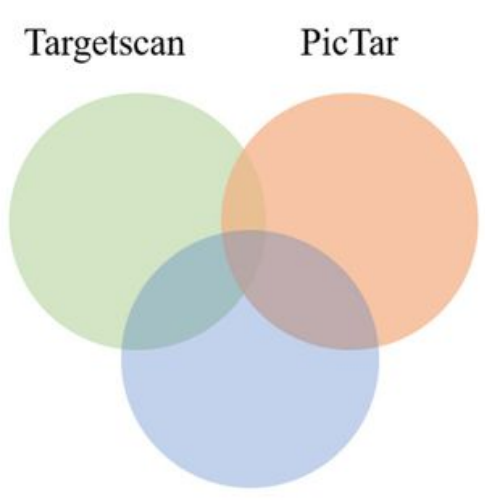

DIANA
PTEN

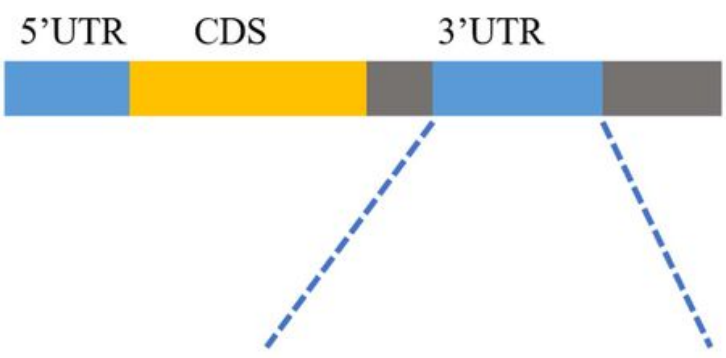

PTEN WT 3'UTR 5'...TACTATTGTAAAGCTAATGTGAA

\|III| miR-23a-3p 3'...CCUUUAGGGACCGUUACACUA

PTEN Mut 3'UTR 5'...TACTATTGTAAAGCTTTACACTA

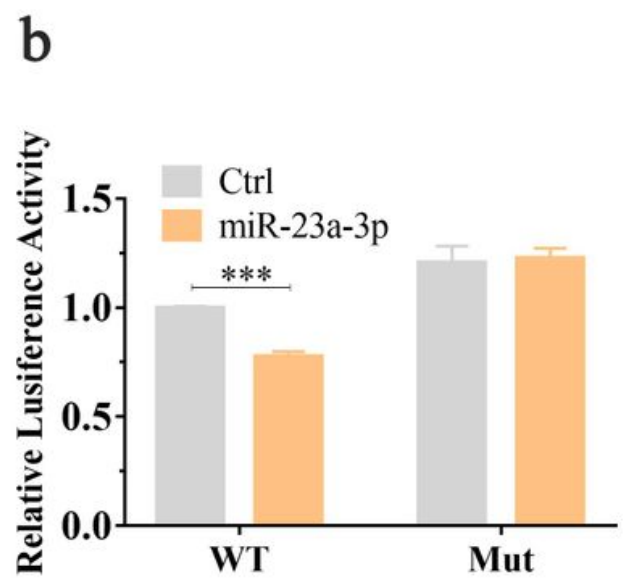

C

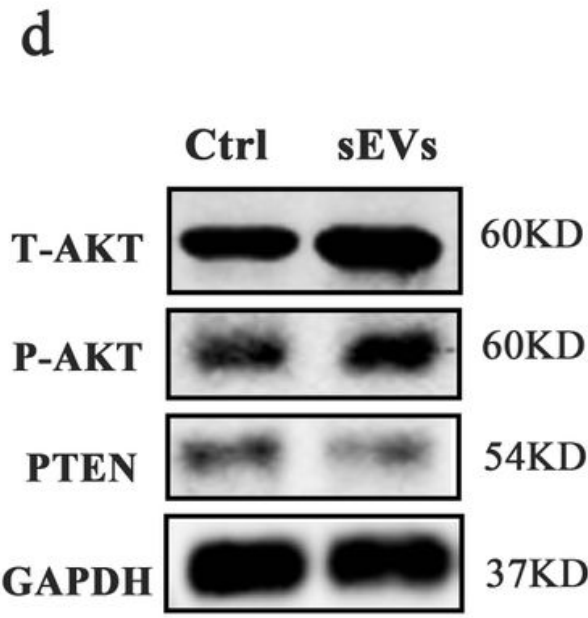

\section{Figure 4}

Exosomal miR-23a-3p regulated the expression of PTEN and AKT by targeted 3'UTR of PTEN. (a) The potential target sequence of miR-23a-3p was predicted by microRNA-target-predicting programs. (b) Luciferase reporter assay was carried out to demonstrate that PTEN is the target gene of miR-23a-3p. (c) BMSCs were cultured with sEVs for $24 \mathrm{~h}$, then qPCR was used to measure miR-23a-3p expression level in BMSCs. Three independent replicates have been statistical analysis. ${ }^{* *} p<0.01$. (d)Western blot was implemented to evaluate the expression levels of AKT, P-AKT, PTEN in BMSCs. 

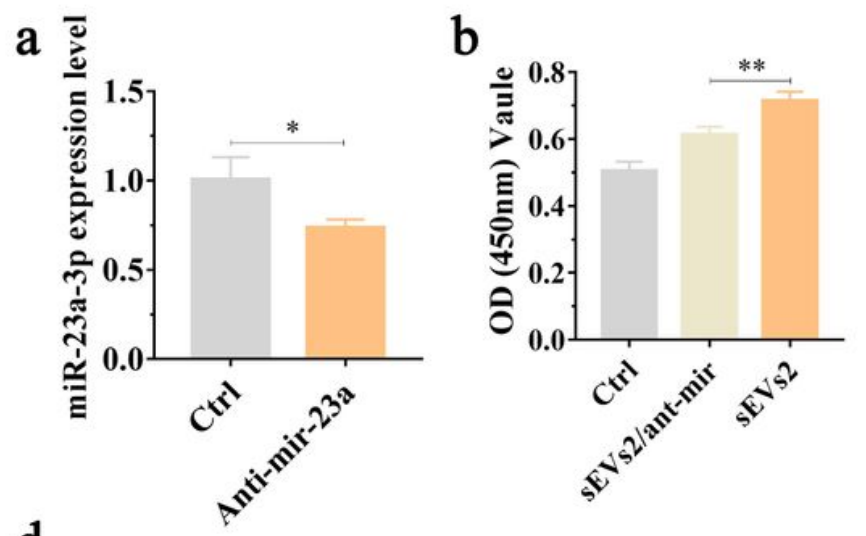

c

d

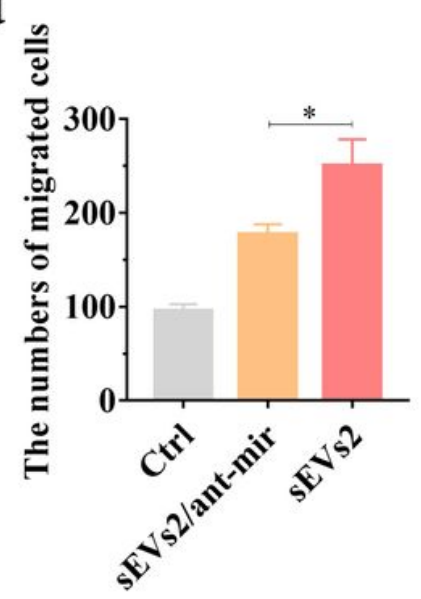

g

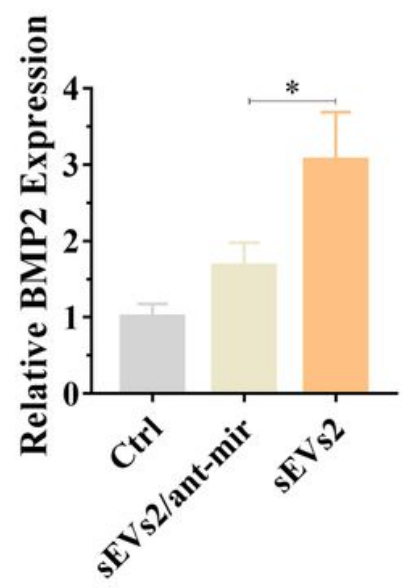

e

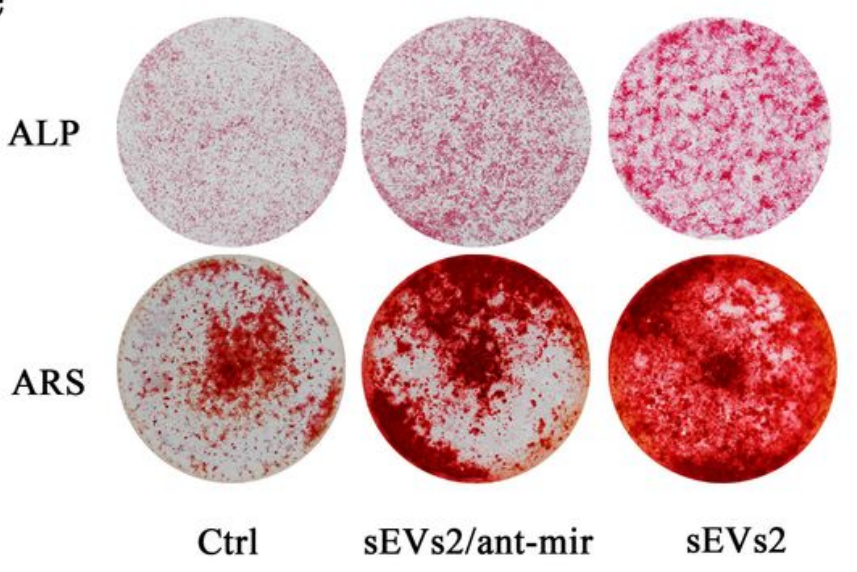

$\mathrm{h}$

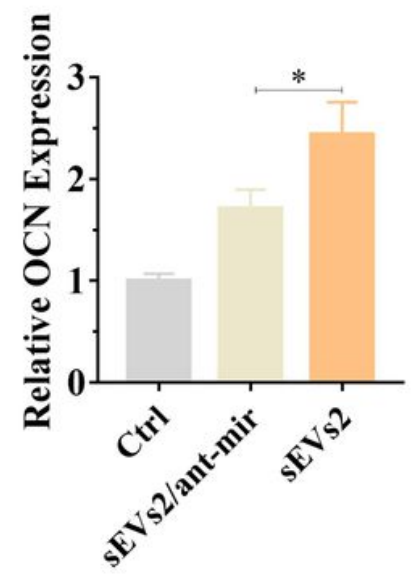

i

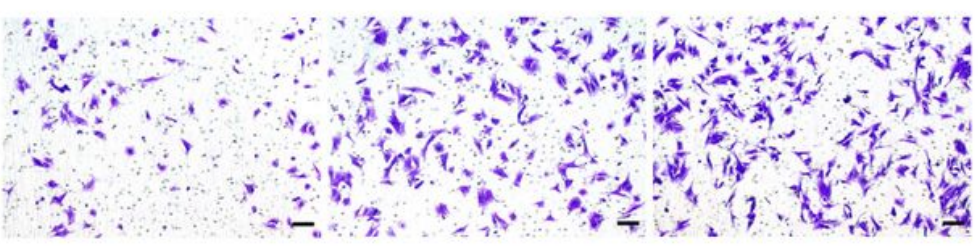

Ctrl sEVs2/ant-mir sEVs2

f

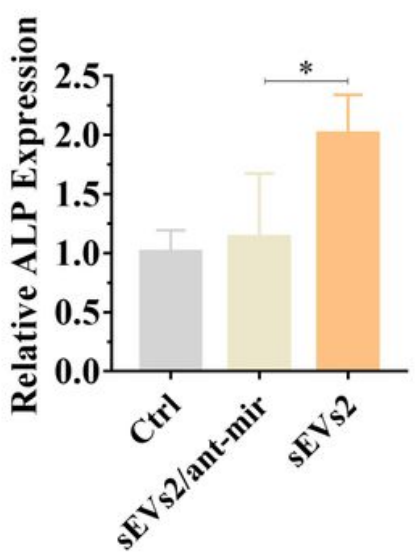

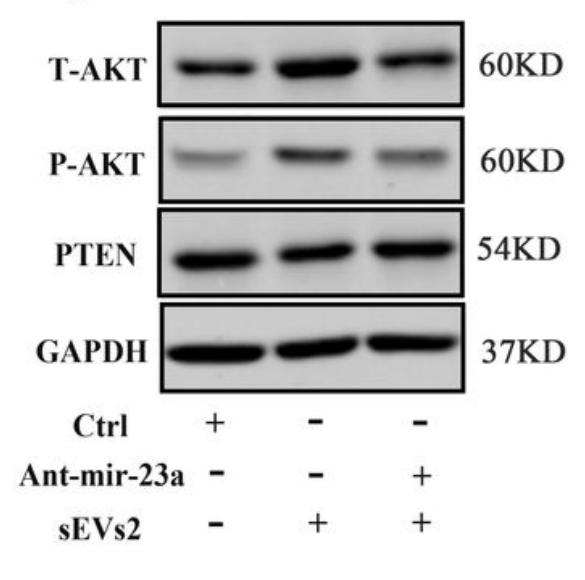

Figure 5

miR-23a-3p silencing alleviated hUC-MSCs-sEVs-mediated proliferation, migration and osteogenic differentiation of BMSCs in vitro by inhibiting PTEN/AKT signaling pathway. (a) BMSCs treated with sEVs were stimulated by antagomir-23a-3p or scramblemir (Ctrl) for $6 \mathrm{~h}$, then qPCR was used to measured miR23a-3p expression level. Three independent replicates have been statistical analysis. ${ }^{\star \star P} P<0.01$. (b) Decreased cell proliferation in BMSCs after administration by antagomir-23a-3p and sEVs. Group sEVs2/ant-mir meant the applied of antagomir-23a-3p and hUC-MSCs-sEVs and group Ctrl meant scramblemir.Three independent replicates have been statistical analysis. ${ }^{*} \mathrm{P}<0.01$. (c) Transwell assay 
of BMSCs which were cultured with scramblemir (Ctrl), sEVs2/ant-mir and sEVs2. (d) Quantitative analysis of transwell assay. Three independent replicates have been statistical analysis. ${ }^{*}<<0.05$. (e) Alkaline phosphatase (ALP) activity and Alizarin Red S (ARS) staining of BMSCs which were cultured with scramblemir (Ctrl), sEVs2/ant-mir and sEVs2. (f-h) Measurement of expression levels of the osteogenic-related gene of BMSCs cultured with scramblemir (Ctrl), sEVs2/ant-mir and sEVs2 via the qPCR, including, ALP, BMP-2 and OCN. (i)Western blotting assay of PTEN/AKT signaling pathway protein including T-AKT, P-AKT and PTEN.
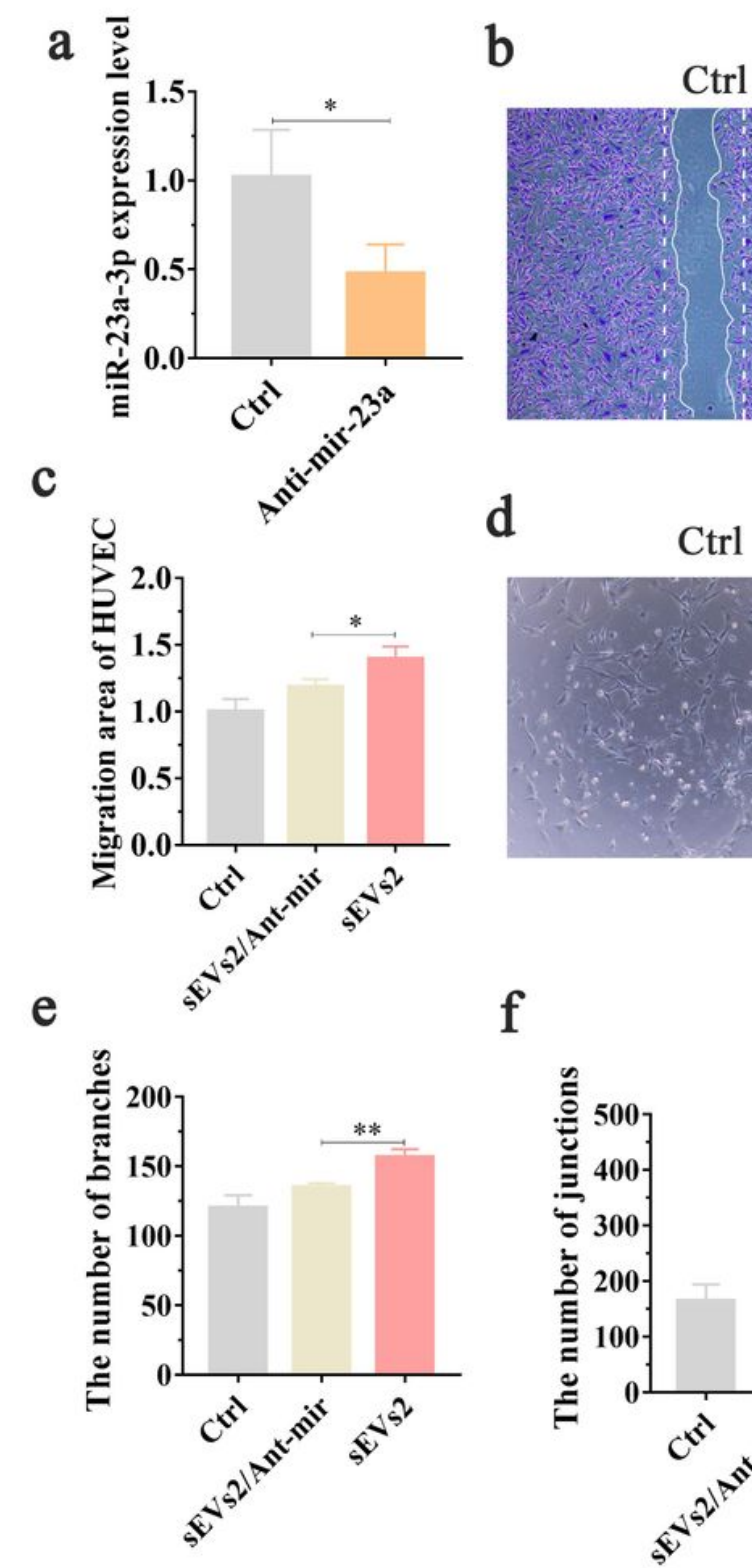

sEVs2/ant-mir
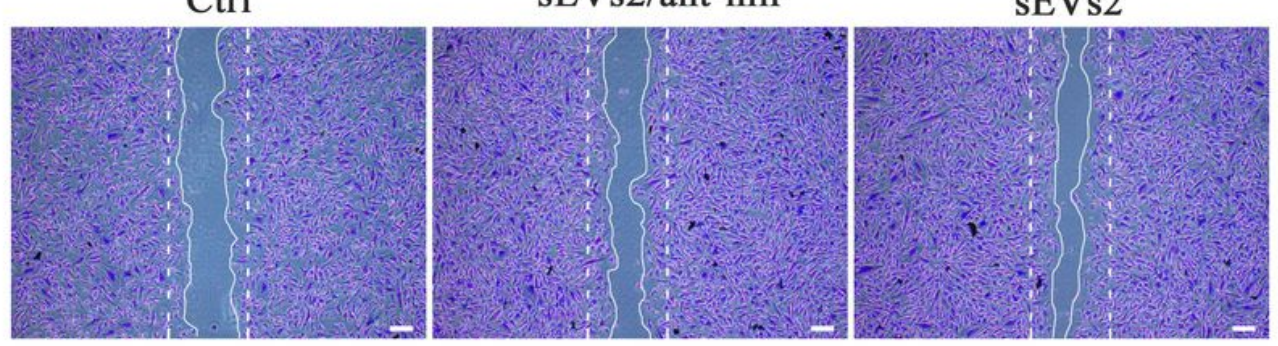

d

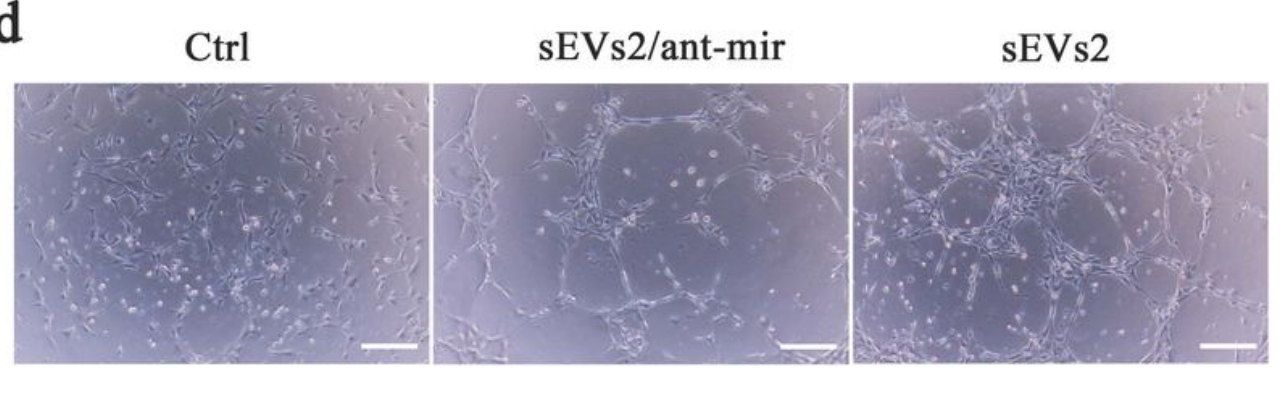

f

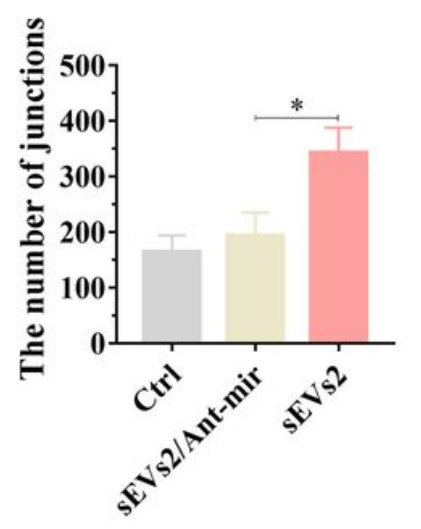

g

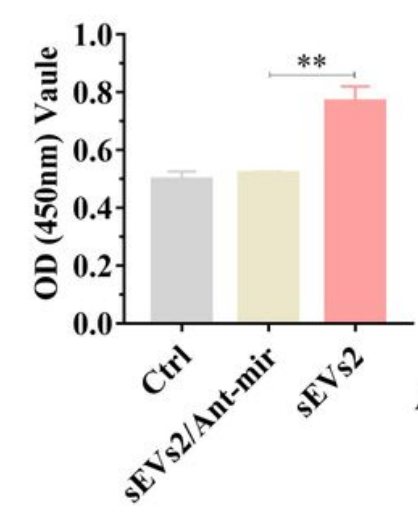

h

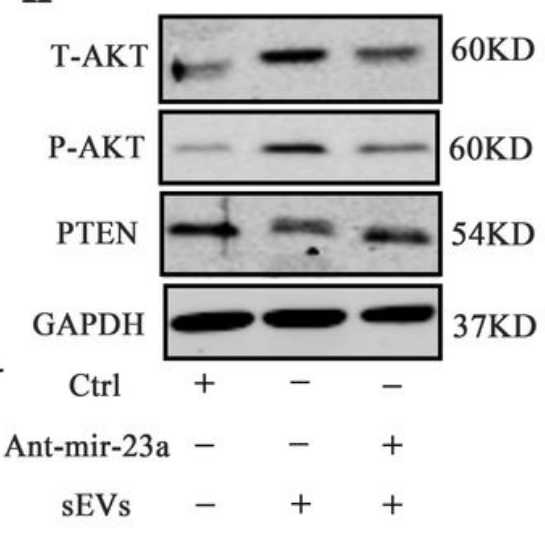

Figure 6

miR-23a-3p silencing alleviated hUC-MSCs-sEVs-mediated pro-angiogenic effectin vitro. (a) HUVECs treated with sEVs were cultured with antagomir-23a-3p or scramblemir (Ctrl) for $6 \mathrm{~h}$, andthen qPCR was used to measured miR-23a-3p expression level. *P $<0.05$. (b)Representative images of "wound healing" 
assay of HUVECs after $8 \mathrm{~h}$ co-culture with scramblemir (Ctrl), antagomir-23a-3p and hUC-MSCs-sEVs, and (c) Quantitative analysis of the relative migrated percentage of HUVECs in different groups. (d) Matrigelbased assay for HUVECs cultured withscramblemir (Ctrl), antagomir-23a-3p and hUC-MSCs-sEVs. Scale bar: 100um. (e)Nb branches and (f) Nb junctions, were all analyzed about tube formation assay by Image J. (g) CCK-8 assay of BMSCs cultured withscramblemir (Ctrl), antagomir-23a-3p and hUC-MSCs-sEVs. **P $<0.01$. (h)Western blotting assay of PTEN/AKT signaling pathway protein including T-AKT, P-AKT and PTEN. Group Ctrl and sEVs2 meant scramblemir and $10 \times 10^{8}$ particles/mL hUC-MSCs-sEVs, respectively.

$\mathbf{a}$

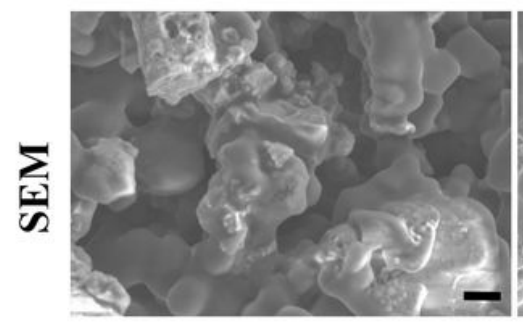

BG

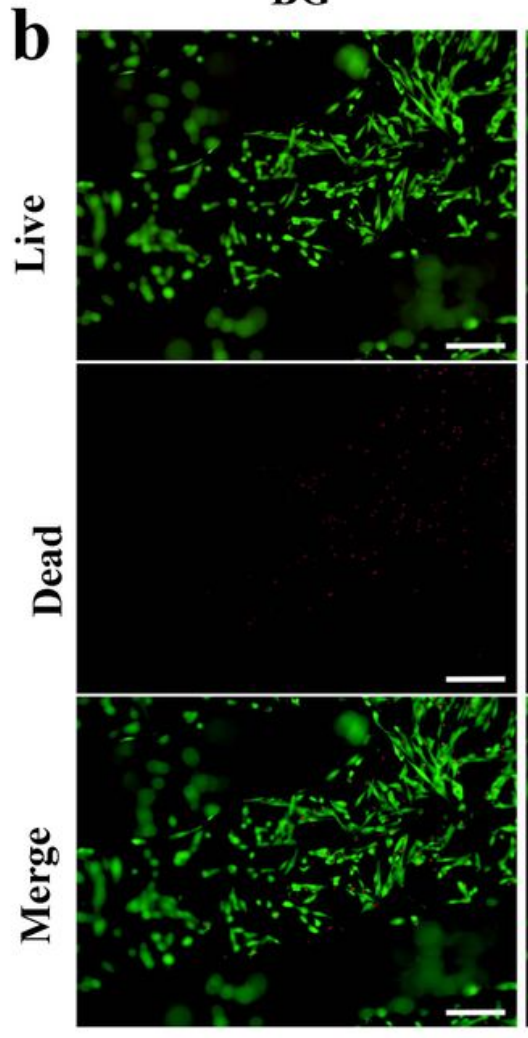

BG-gel

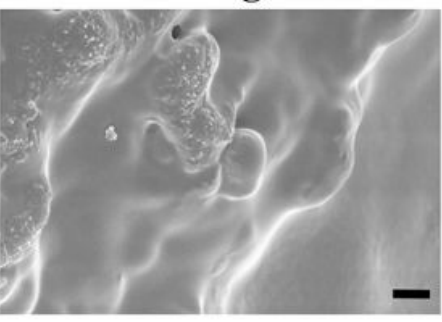

BG-gel

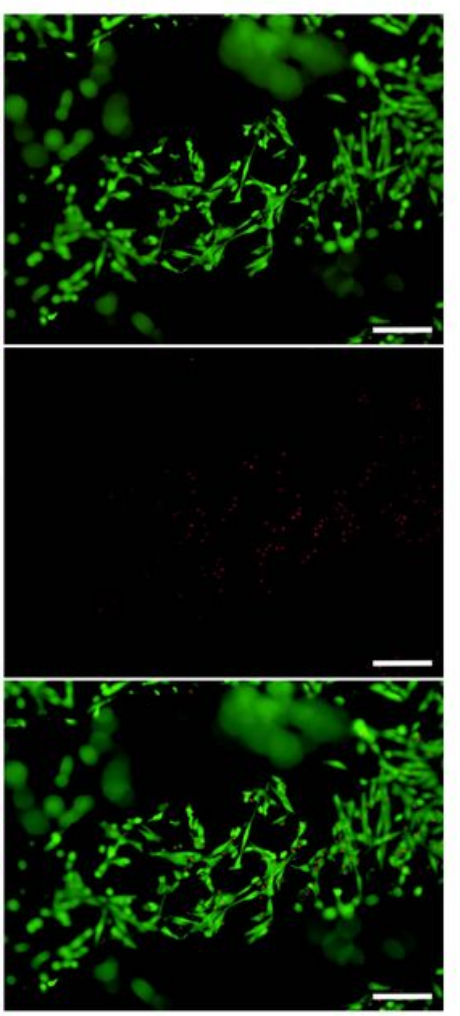

C BG-sEVs
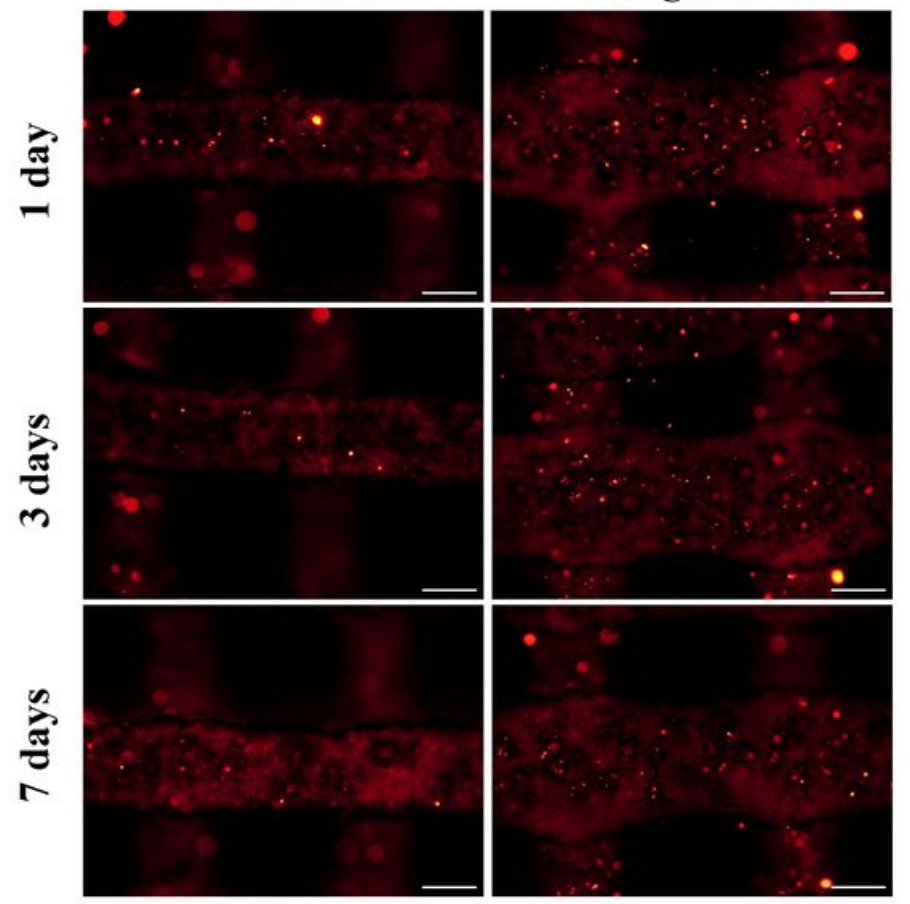

d

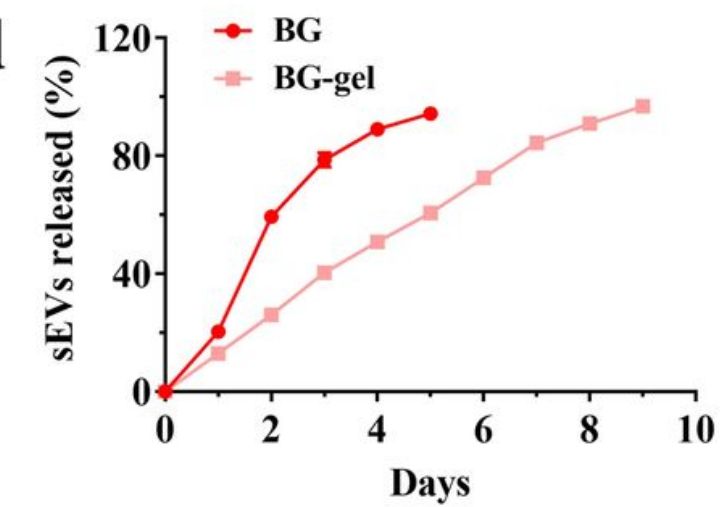

Figure 7

Characterization of engineered BG composited scaffolds. (a) Scanning electron micrographs of BG, BGgel scaffolds. Scale bars, $2 \mu \mathrm{m}$. (b) Fluorescence images of BMSCs stained with calcein AM (live cells

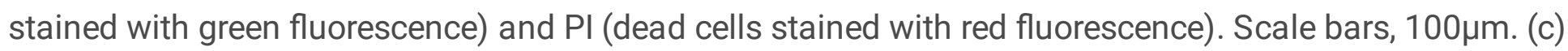

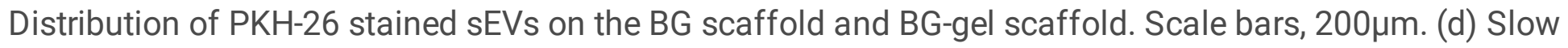
release profile of sEVs loaded by BG-gel scaffold. 
a
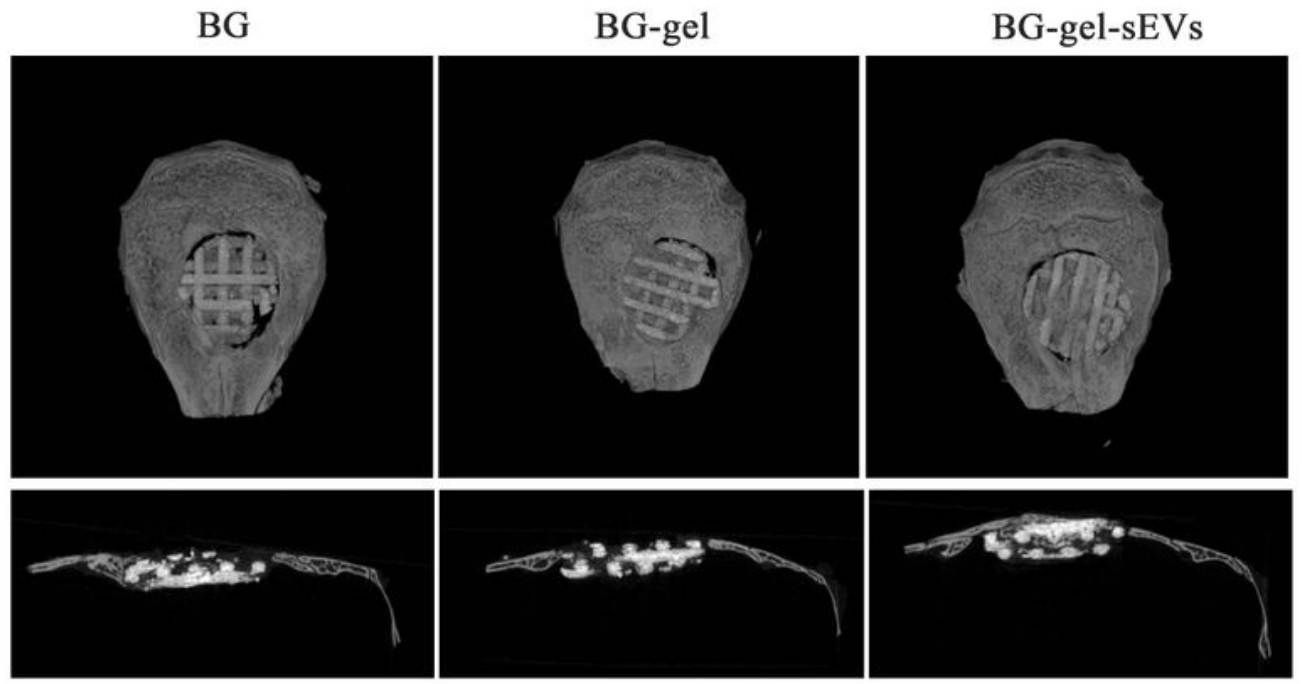

$\mathrm{b}$

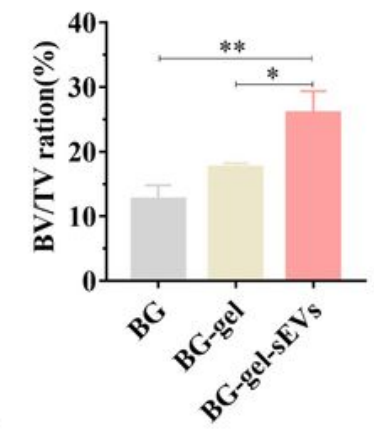

c
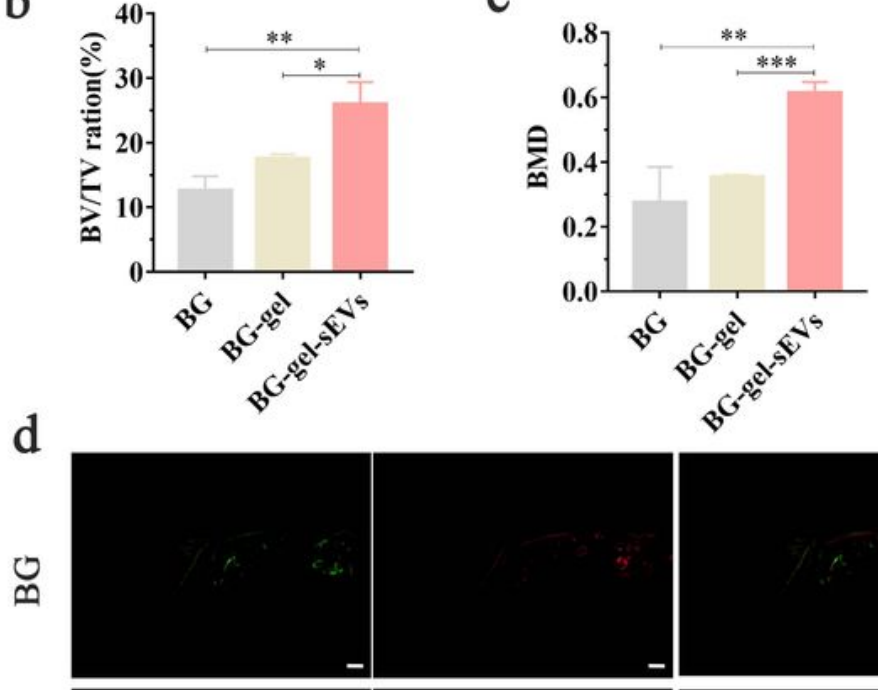

e
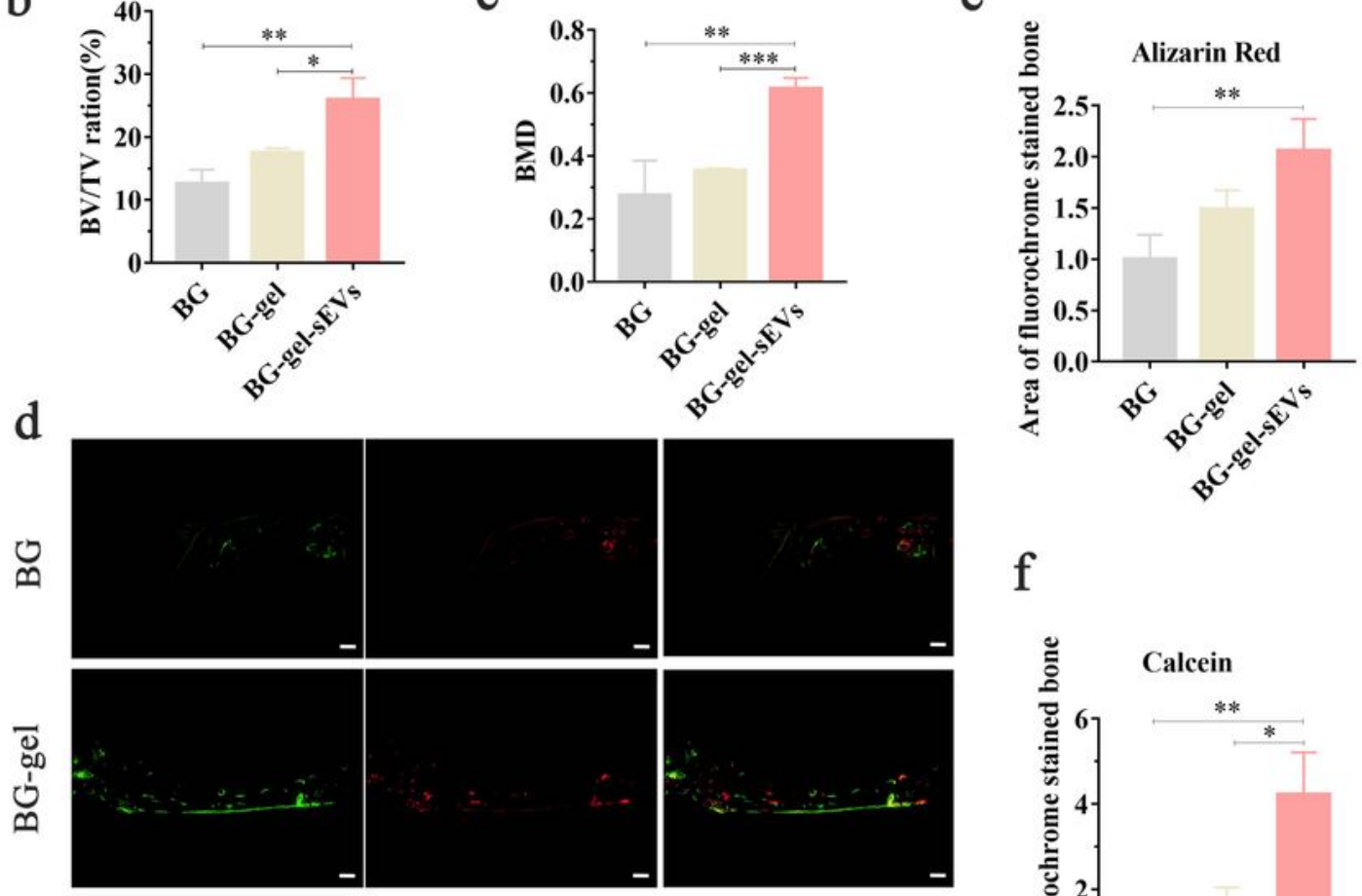

f
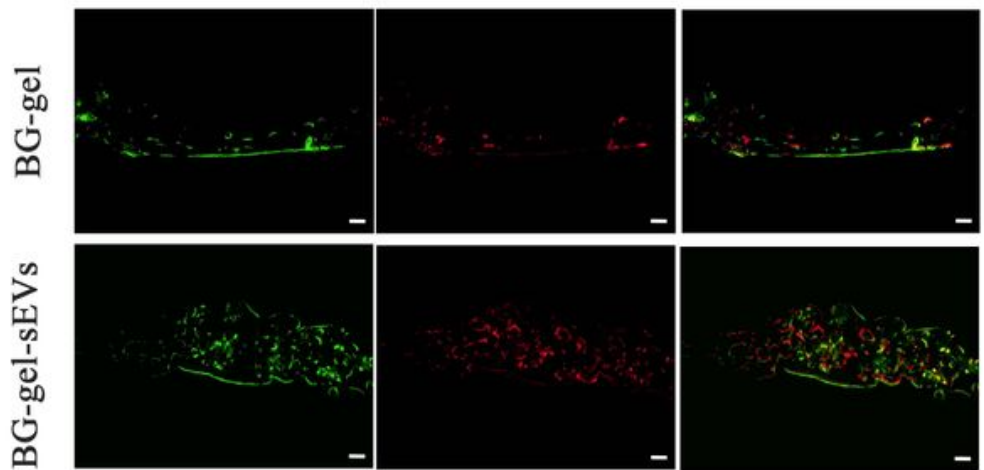

Calcein

Alizarin Red

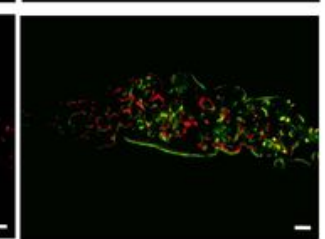

Merge

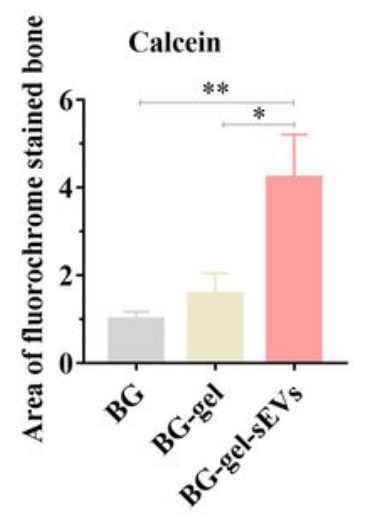

\section{Figure 8}

Micro-CT and morphometric assessment of calvarial defect repaired by BG-gel-sEVs scaffold. (a) Representative coronal and sagittal micrograph of bone defect. (b-c) Quantitative analysis of the new bone including bone volume/tissue volume (BV/TV), bone mineral density (BMD). ${ }^{*} P<0.05,{ }^{*} P<0.01$, $\star \star \star P<0.001$. (d) Sequential fluorescent labeling of the regenerated bone by calcein and alizarin red. Scale 
bar: 200um. (e-f) Quantitative analysis of each dye area for different scaffolds, and the results of BG group was classified as one. ${ }^{\mathrm{P}}<0.05,{ }^{\star *} \mathrm{P}<0.01$.
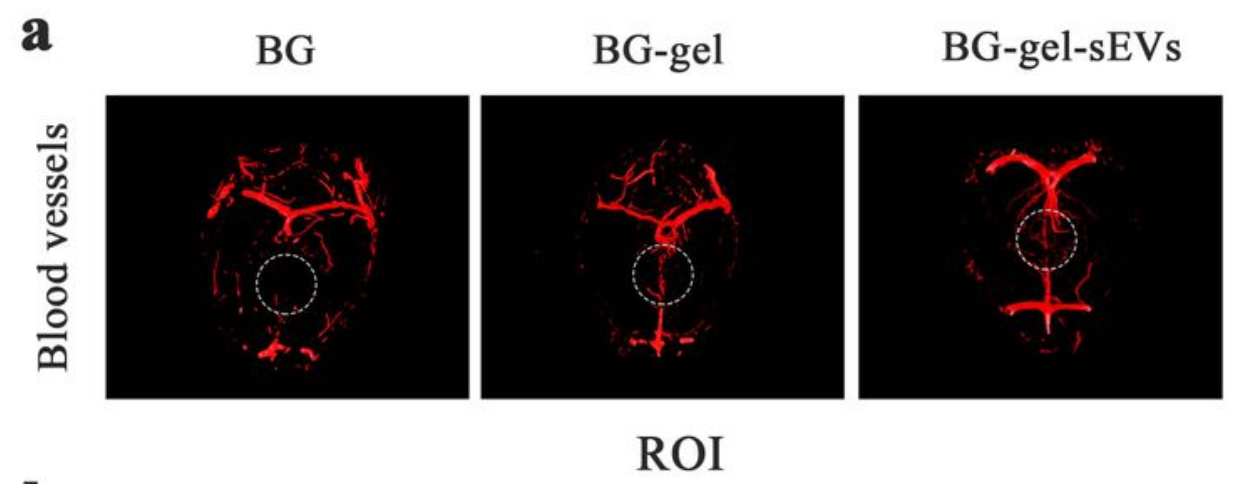

b
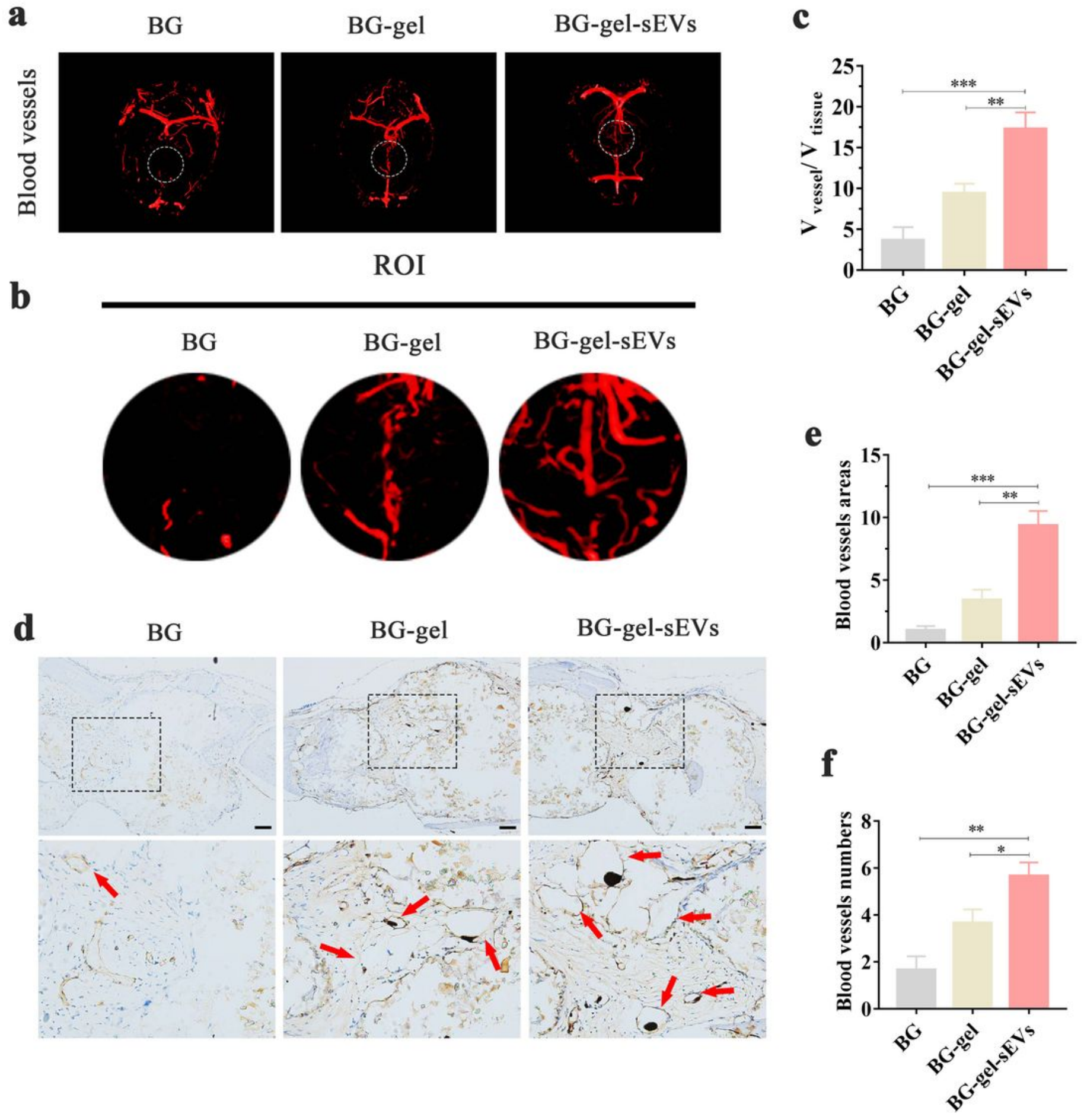

Figure 9

Effect of BG-gel-sEVs scaffold on vascularization in vivo.(a) Regenerated blood vessels (within the white circle) scanned by Micro-CT in the defect regions repaired by different scaffolds. (b) Regenerated blood 
vessels in regions of interest (ROI) selected for measuring the blood vessel volume. (c)Quantitative analysis for regenerated blood vessels. ${ }^{*} \mathrm{P}<0.01$, ${ }^{\star * *} \mathrm{P}<0.001$. (d) $C D 31$ immunohistochemical staining was used to observe the newly formed blood vessels in the defect regions. The quantitative analysis of blood vessels area (e) and number (f). ${ }^{*} P<0.05,{ }^{*} \mathrm{P}<0.01,{ }^{*} * \mathrm{P}<0.001$.
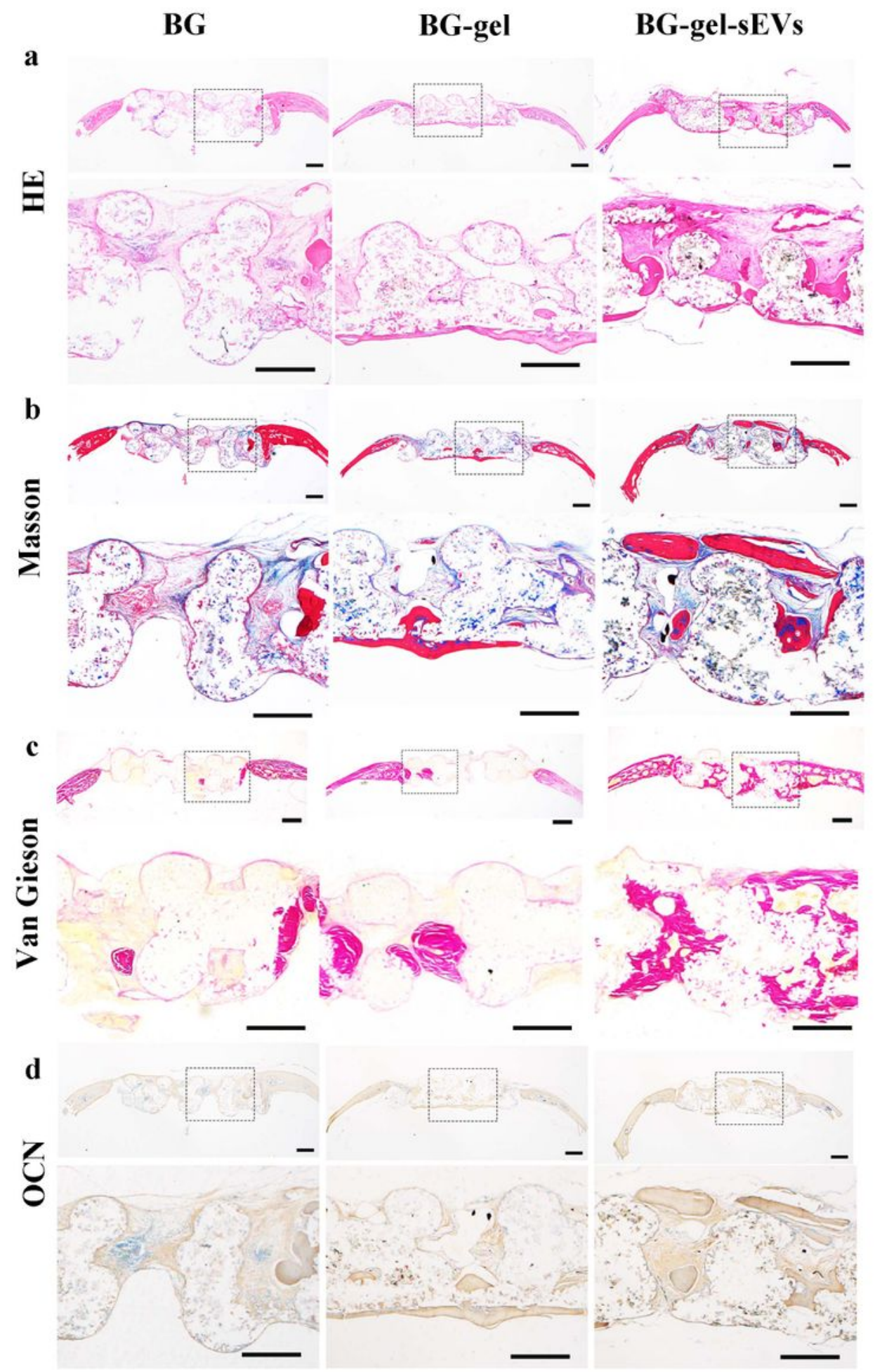

Figure 10 
Histological evaluation of calvarial bone defects repaired by BG-gel-sEVs scaffolds. (a) HE staning and (b)Masson's trichrome staining of decalcified bone samples repaired by different scaffolds. Scale bar: 500um. (c) Van Gieson's staining of regenerated bone repaired by different scaffolds. Scale bar: 500um. (d)Immunohistochemical staining of OCN. Scale bar: 500um.

\section{Supplementary Files}

This is a list of supplementary files associated with this preprint. Click to download.

- graphicabstract.tif

- Supplementarymaterials.docx

- scheme1.png 